\title{
OPEN Probing natural variation of IRE1 expression and endoplasmic reticulum stress responses in Arabidopsis accessions
}

\author{
Taiaba Afrin, Minye Seok, Brenna C. Terry \& Karolina M. Pajerowska-Mukhtar ${ }^{\bigotimes}$
}

The environmental effects shape genetic changes in the individuals within plant populations, which in turn contribute to the enhanced genetic diversity of the population as a whole. Thus, individuals within the same species can acquire and accumulate genetic differences in their genomes depending on their local environment and evolutionary history. IRE1 is a universal endoplasmic reticulum (ER) stress sensor that activates an evolutionarily conserved signalling cascade in response to biotic and abiotic stresses. Here, we selected nine different Arabidopsis accessions along with the reference ecotype Columbia-0, based on their geographical origins and differential endogenous IRE1 expression under steady-state conditions to investigate the natural variation of ER stress responses. We cloned and analysed selected upstream regulatory regions of IREI $a$ and IRE1b, which revealed differential levels of their inducibility. We also subjected these accessions to an array of biotic and abiotic stresses including heat, ER stress-inducing chemical tunicamycin, phytohormone salicylic acid, and pathogen infection. We measured IRE1-mediated splicing of its evolutionarily conserved downstream client as well as transcript accumulation of ER-resident chaperones and co-chaperones. Collectively, our results illustrate the expression polymorphism of a major plant stress receptor and its relationship with molecular and physiological ER stress sensitivity.

Because of their sessile nature, plants constantly need to respond to their surrounding environment and adapt themselves to the ever-changing conditions to ensure a suitable balance for growth and survival ${ }^{1}$. Thus, the native habitat imposes on species a pressure to survive and evolve along with environmental changes ${ }^{2}$. The multidimensional climate fluctuations trigger simultaneous genetic variations in the individuals within the plant population, contributing to an increase in the genetic diversity of the population as a whole ${ }^{3}$. Therefore, the individuals of the same species can exhibit distinct variation in their genome sequences depending on their geographical origins and evolutionary history. The genetic variation found in populations from different natural environments demonstrates the extent of local adaptation ${ }^{4}$ and allows the discovery of novel genes and alleles as signatures of plants' adaptive responses ${ }^{5}$. As such, studying natural variation can provide important insights into diverse structural and functional features: novel gene and allele identification ${ }^{6}$, cause and effect of phenotypic variation ${ }^{7}$, understanding complex traits and their impact on phenotypes ${ }^{8}$, and selective pressure towards specific traits ${ }^{8}$. These discoveries can also be useful to engineer agronomically important crop plants for better compatibility with the changing climate. Arabidopsis thaliana (hereafter Arabidopsis) originates from continental Eurasia and North Africa but is now extensively distributed throughout the world ${ }^{9}$. Its natural habitat is widely diversified, from beaches to the Rocky Mountains, riverbanks to roadsides ${ }^{4,9,10}$. The broad spectrum of Arabidopsis natural habitats is a major contributor to its substantial genetic variation ${ }^{11}$. Arabidopsis natural accessions around the globe show considerable genetic and phenotypic variation in terms of plant development, physiology, and adaptation to biotic as well as abiotic stresses, manifested through traits such as rosette diameter ${ }^{12}$, plant height ${ }^{13}$, number of lateral branches $^{13}$, leaf shape ${ }^{12}$, flowering time ${ }^{14}$, the structure of inflorescence ${ }^{15}$, seed dormancy ${ }^{16}$, drought resistance ${ }^{6}$, heat tolerance ${ }^{17}$, cold tolerance ${ }^{18}$, salt tolerance ${ }^{11}$, disease resistance ${ }^{19}$, resistance and tolerance to herbivores ${ }^{20}$, and circadian rhythms ${ }^{21}$. Owing to its short life cycle and small, fully sequenced genome, Arabidopsis has been at the forefront of plant model systems for the last 35 years $^{22}$. Its worldwide distribution ${ }^{9,23}$, rich genetic resources ${ }^{9,23}$, feasibility to maintain pure lines ${ }^{9}$, adaptive nature ${ }^{23}$, availability of genome-wide single nucleotide polymorphism $(\mathrm{SNP})^{4,9}$, and collections of materials developed by the international community ${ }^{16}$ further increased its 
usefulness as the model plant. The analysis of Arabidopsis natural variation has the potential to equip us with a unique understanding of functional, ecological and evolutionary connections and reationships ${ }^{8}$. In 1996, the Arabidopsis Genome Initiative (AGI), an international collaborative community, initiated a project to sequence the Arabidopsis genome ${ }^{24}$ and in 2008 followed up with a large-scale effort known as the "1001 Genome Project" to provide more refined genetic tools to the Arabidopsis community ${ }^{25}$. For instance, a comparative study reported that the two most-used and highly related ${ }^{22}$ Arabidopsis strains, Columbia-0 (Col-0) and Landsberg erecta (Ler) differ by a total 25,274 SNPs in their coding and non-coding regions ${ }^{24}$, which underscores the abundance of genetic natural variation within Arabidopsis accessions.

The endoplasmic reticulum (ER), the largest membrane system in eukaryotic cells, plays crucial roles in a variety of cellular processes, i.e. synthesis of membrane proteins, membrane lipids, secretory proteins, protein folding, glycosylation, disulfide bonding, post-translational modifications, and packaging to target location ${ }^{26,27}$. Disturbances or malfunctions in any of these processes result in the accumulation of malfolded and/or unassembled proteins and subsequently trigger ER stress. The mechanisms of ER stress signalling have been studied extensively in yeast ${ }^{28}$, mammals ${ }^{27,28}$, and plants ${ }^{29-31}$. In plants, ER stress can be established by application of specific treatments, i.e. chemicals (tunicamycin; $\mathrm{Tm}^{32,33}$, dithiothreitol; DTT ${ }^{31,34}$, salicylic acid; $\mathrm{SA}^{33,35}$, L-azetidine-2-carboxylic $\operatorname{acid}^{36}$, cyclopiazonic acid; $\mathrm{CPA}^{33}$ ), viral and bacterial pathogens ${ }^{33,37,38}$, heat stress ${ }^{35,39,40}$, and salt stress ${ }^{41}$, as well as during normal growth and developmental process ${ }^{31,37,42}$. ER stress elicits several cellular responses, with the unfolded protein response (UPR) playing the predominant role ${ }^{32,43}$. UPR is a complex eukaryotic signalling pathway that functions to restore cellular homeostasis ${ }^{28-30}$. The key UPR signal activator and ER stress sensor, Inositol-Requiring Enzyme 1 (IRE1), is evolutionarily ancient and highly conserved in eukaryotes $^{43}$. In Arabidopsis, two homologues of IRE1, known as IRE1a and IRE1b, are the critical players in the UPR signalling pathway ${ }^{32,33,44}$. IRE $1 a$ and IRE1b genes share $41 \%$ nucleotide sequence similarity and exhibit both overlapping and distinct expression patterns ${ }^{32,44,45}$. While both of these isoforms are expressed throughout the plant under steady-state conditions, IRE1b is specifically enriched in embryos and seeds ${ }^{32,44}$ and was reported to be essential for functional male fertility ${ }^{46}$. Under stress-induced conditions, however, the two homologues show more profound functional divergence. IRE1a plays a predominant role in biotic stresses ${ }^{33}$, while IRE1b is critical during abiotic stresses ${ }^{39}$, pointing towards genetic and physiological specialisation and diversification of the two IRE1 isoforms. How the IRE1 homologues were shaped by the evolutionary forces in diverse Arabidopsis accessions to mitigate the ER stress is an intriguing question.

Structurally, the IRE1 proteins possess well-conserved serine/threonine protein kinase and endoribonuclease (RNase) domains, which allow IRE1 to perform dual functions ${ }^{47}$. After sensing ER stress, IRE1 dimerises, undergoes trans-autophosphorylation, and transduces downstream UPR signalling. In Arabidopsis, IRE1a and IRE1b recognise splice-site motifs in the transcript of an evolutionarily conversed basic leucine zipper transcription factor bZIP60 and catalyse an unconventional cytoplasmic mRNA cleavage. This processed (spliced) form of bZIP60 mRNA undergoes translation, producing an active protein that translocates to the nucleus and transcriptionally regulates an array of UPR-responsive genes to exert a cytoprotective function ${ }^{31,38,48}$. The IRE1/ bZIP60 signalling pathway plays a distinct role in mitigating both biotic and abiotic stresses to restore cellular homeostasis $^{33,49}$. This unconventional splicing is referred to as regulated IRE1-dependent splicing (RIDS). Under acute or prolonged ER stress, IRE1 also degrades mRNAs through a site-specific cleavage process termed as regulated-IRE1 dependent RNA decay (RIDD) ${ }^{50,51}$. In Arabidopsis, RIDD mainly targets the mRNAs encoding secreted proteins ${ }^{52}$.

Abiotic and biotic stress factors have been shown to activate the ER stress signalling in plants. Among several environmental stressors, heat has been previously identified as a major factor affecting the vegetative and reproductive growth of plants $s^{53,54}$. Heat is also known to be a powerful inducer of UPR in yeast ${ }^{55}$, mammals ${ }^{56}$, and plants $33,35,39,40,57,58$. Upon heat stress, plant cells initiate a cascade of stress responses, including the UPR signalling in the $\mathrm{ER}^{55,56}$. The phytohormone salicylic acid (SA) plays a pivotal role in several growth and developmental processes ${ }^{59,60}$, disease resistance signaling ${ }^{61,62}$, and defence responses against biotrophic pathogens ${ }^{63,64}$. Moreover, SA was previously shown to activate the IRE1/bZIP60 arm of the UPR signalling pathway via $b Z I P 60$ splicing ${ }^{58,65,66}$. Here, we selected 10 representative natural accessions of Arabidopsis and studied the contributions of genetic variation of IREI $a$ and IRE $1 b$ and downstream ER stress responses in the context of biotic and abiotic triggers. We showed that both IREIa and IRE1b vary in their expression levels in the set of selected Arabidopsis ecotypes, and we demonstrated differential levels of $b Z I P 60$ splicing in diverse ecotypes in response to SA and heat. We also detected varied accumulation levels of UPR chaperons and co-chaperons upon SA treatment. Finally, we evaluated whole-plant tolerance of the accessions to ER stress triggered by heat and Tm as well as disease resistance phenotypes of these accessions upon infection with a bacterial pathogen. Overall, we provided insights into the natural variation of ER stress responses in Arabidopsis.

\section{Results and discussion}

Selection of the representative accessions to study genetic variation of IRE1a and IREIb expression. To better understand how the evolutionary forces and natural selection have shaped the regulatory regions of IRE $1 a$ and IRE $1 b$ loci in Arabidopsis, we studied Col-0 as the reference accession along with nine additional accessions stemming from six different countries (Fig. 1, Table 1). Our selection was based on the values of basal expression levels of IRE1a and/or IRE1b genes in respective accessions, derived from the Plant eFP browser ${ }^{67-70}$. Specifically, we selected Bla-5, Dra-1 and En-T as accessions that display the highest basal expression of IRE1 a compared to Col-0, while Is- 0 and M7323S were the additional two accessions with the lowest basal IRE1a transcript levels (Table 1). We used the same strategy for IRE1b and selected M7323S, HR-5, and Fr-2 that exhibited elevated basal expression levels, and MS-0 and Ta- 0 that were characterised by diminished basal IRE1b transcript accumulation compared to Col-0. 


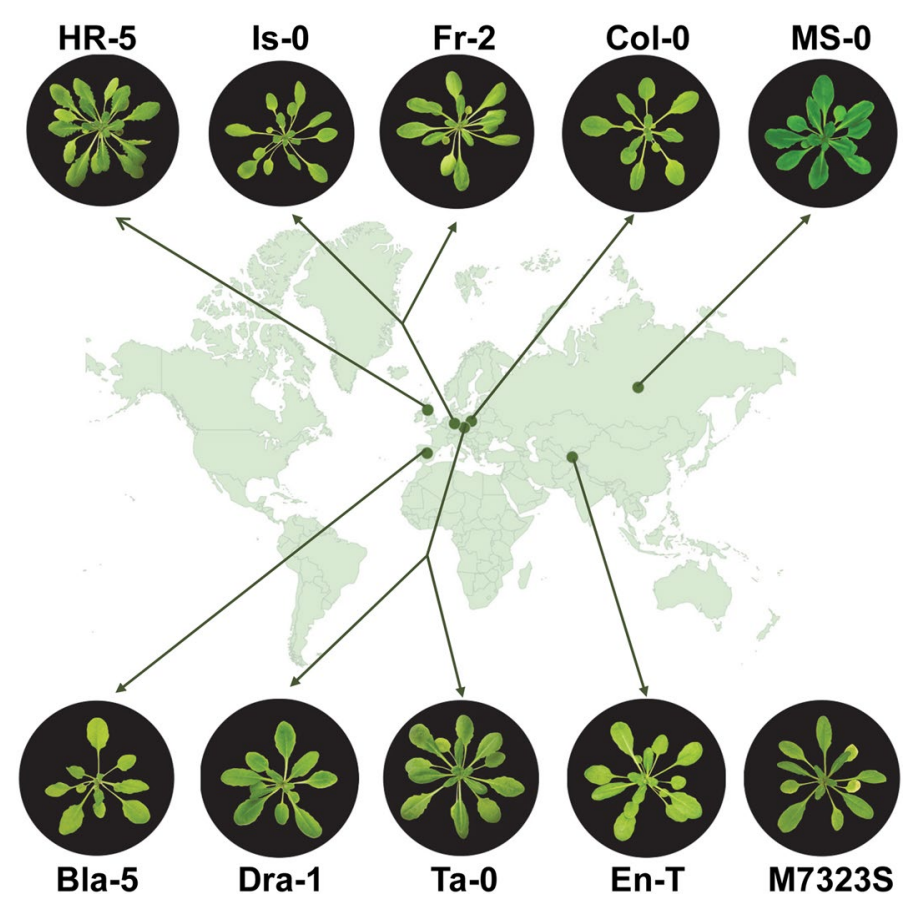

Figure 1. Geographical distribution and representative phenotypes of selected Arabidopsis natural accessions used in this study. Geographical origins are indicated by green dots on the world map. The origin of M7323S is unknown. 1-month-old plants representative of ecotypes Col-0, Bla-5, Dra-1, En-T, Is-0, MS-0, M7323S, HR-5, Fr-2, and Ta- 0 are pictured. Plants representing individual accessions were photographed by NIKON D5600 camera. A world map background was generated using Google Sheets (a free resource). The figure was finalized using Adobe Photoshop (Version: 21.2.4).

\begin{tabular}{|c|c|c|c|c|c|c|c|}
\hline Accessions for IRE1a & $\begin{array}{l}\log 2 \\
\text { Ratio }\end{array}$ & $\begin{array}{l}\text { Fold } \\
\text { Change }\end{array}$ & Country of Origin & Accessions for IREIb & $\begin{array}{l}\log 2 \\
\text { Ratio }\end{array}$ & \begin{tabular}{|l} 
Fold \\
Change
\end{tabular} & Country of Origin \\
\hline Col-0 & 0.0 & 1.0 & NW Poland & Col-0 & 0.0 & 1.0 & NW Poland \\
\hline Bla-5 & 0.7 & 1.62 & Blanes, Spain & MS-0 & 0.32 & 0.8 & Moscow, Russia \\
\hline Dra-1 & 0.79 & 1.73 & $\begin{array}{l}\text { Drahonin, Czech } \\
\text { Republic }\end{array}$ & M7323S & 0.34 & 1.27 & Unknown \\
\hline En-T & 1.12 & 2.17 & Tajikistan & HR-5 & 0.29 & 1.22 & United Kingdom \\
\hline Is-0 & -0.86 & 0.55 & Isenberg, Germany & Fr-2 & 0.46 & 1.38 & Frankfurt, Germany \\
\hline M7323S & -0.81 & 0.57 & Unknown & Ta-0 & -0.32 & 0.8 & Tabor, Czech Republic \\
\hline
\end{tabular}

Table 1. Selected accessions for IRE1 $a$ and $I R E 1 b$ genes, characterised in this study. Basal expression log 2 ratios and fold changes (derived from Arabidopsis eFP browser) as well as geographical origin, if known, are shown.

Differential response of IREIa and IREIb genes to heat stress. We set out to validate the basal expression values obtained from the Plant eFP browser through an independent experiment. In plants, heat is known to be a powerful inducer of UPR ${ }^{33,35,39,57,58}$; thus, we also examined the inducibility of IRE1 $a$ and IRE1b expression following heat treatment to assess whether basal levels coincide with induced transcript accumulation in both IRE1a-and IRE1b-related groups. Towards this, we exposed our selected natural accessions to a 90-min long treatment of elevated temperature $\left(37^{\circ} \mathrm{C}\right)$. Following heat stress, we collected foliar tissues and quantified both basal and induced levels of IRE1a or IRE1b (Fig. 2a-d). We used an ire $1 a-2$ ire $1 b-4$ double mutant as a negative control in our experiment (Fig. S1a). Our results indicated that the accessions in IRE1a-related group (Bla-5, Dra-1, En-T, Is-0, M7323S) and IRE1b-related group (MS-0, M7323S, HR-5, Fr-2, and Ta-0) showed basal IRE1a transcript accumulation levels comparable to the results reported by the plant eFP browser ${ }^{68}$ dataset (Fig. 2a,b). Specifically, Bla-5, Dra-1, and En-T plants in the IRE1a-related group exhibited higher basal IRE1a transcript levels, whereas Is- 0 and M7323S displayed lower basal IRE1 $a$ accumulation. In the IRE1b-related group, we did not observe any significant change in the IRE1a basal transcript accumulation, consistent with the eFP browser ${ }^{68}$ dataset (Fig. 2b). When we analysed heat-induced IRE1a transcript levels in the IRE1a accession group (Fig. 2a), all ecotypes but Bla-5 significantly induced the IRE1a expression with respect to their basal levels. Subsequently, we compared the IRE1 $a$ induction in these ecotypes with the reference accession Col-0. We demonstrated that 


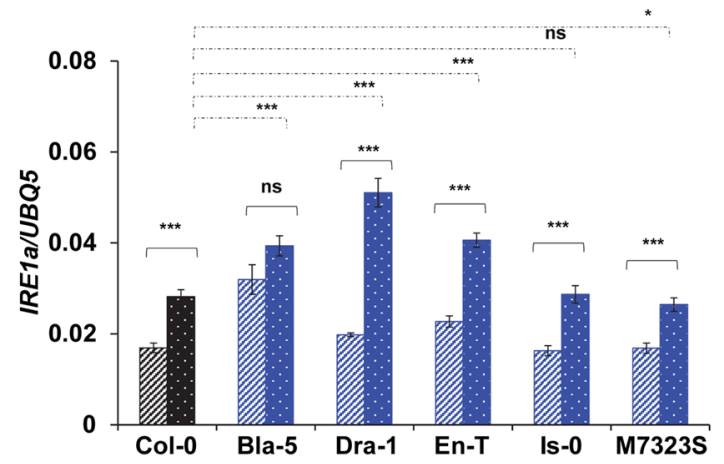

b

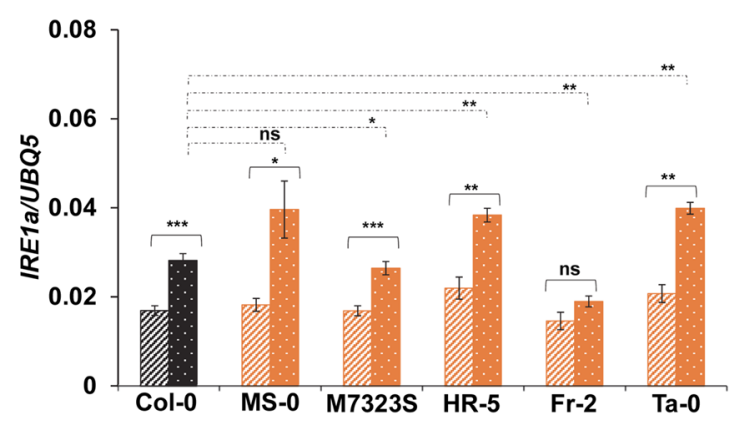

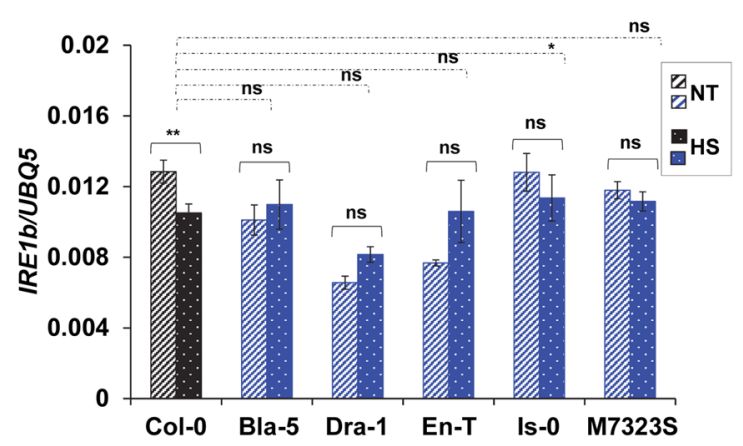

d

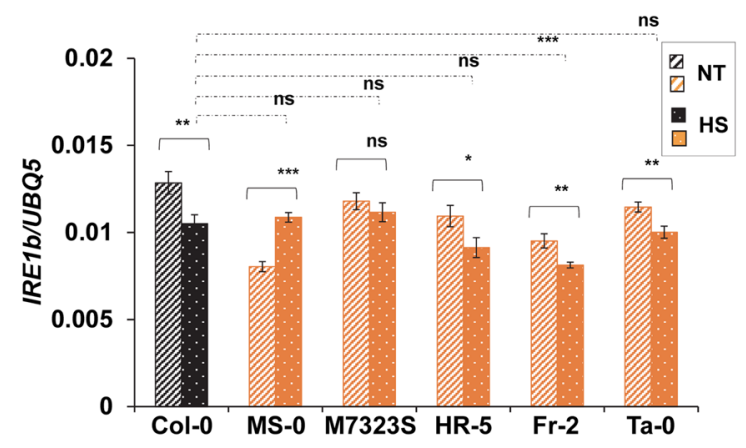

Figure 2. Analysis of relative IRE1 $a$ and $I R E 1 b$ expression levels in selected accessions before and after heat stress. Basal and induced mRNA expression level of $\operatorname{IRE1a}(\mathbf{a}, \mathbf{b})$ or $\operatorname{IRE} 1 b(\mathbf{c}, \mathbf{d})$ of indicated accessions upon heat stress at $37^{\circ} \mathrm{C}$ for $90 \mathrm{~min}$. Expression levels were measured in leaf tissue of 1-month-old Arabidopsis plants via qRT-PCR and were normalised to the housekeeping gene UBQ5 (Ubiquitin 5). Dashed bars represent basal expression levels, dotted bars correspond to heat-induced expression levels. Treatment groups are represented according to legends. Colours indicate accessions grouping (blue-IRE1a-related accessions, orange-IRE1brelated accessions, black-reference accession Col-0). Statistical analyses were performed in Excel by One-Way ANOVA. At least three independent biological replicates, each with three technical replicates were performed. Error bars show mean \pm SD. Significant differences are indicated by asterisks $\left({ }^{\star * *} p<0.001,{ }^{\star *} p<0.01,{ }^{\star} p<0.05\right)$, while "ns" indicates no statistically significant differences. Solid lines connecting bars represent the comparison of basal to heat-induced expression levels for each individual accession, while dashed lines represent the comparison of induced expression levels between Col-0 and an indicated accession.

three ecotypes (Bla-5, Dra-1, and En-T) showed significantly higher IRE1a expression levels compared to Col0, M7323S had a significantly reduced IRE1a expression, while the heat-induced IRE1a expression in Is-0 was comparable to Col-0. In the IRE1b-related group, IRE1a expression was significantly increased in all accessions except Fr-2 when compared to their corresponding controls. We also assayed the heat-induced IRE1a transcript compared to reference accession Col- 0 and demonstrated a modest but statistically significant increase in the levels of expression in all accessions under study except MS-0 (Fig. 2b). When comparing fold induction above the basal levels of each accession, the strongest inducers of IRE1a were Dra-1, MS-0, and Ta-0 (Fig. S1b, c). Taken together, our data indicate that all of the assayed accessions show an intact ability to induce IRE1a following heat stress, and the basal levels are not an accurate predictor of heat inducibility for the Arabidopsis IRE1a gene (Fig. 2a,b).

Next, we tested IRE1b mRNA levels in both IRE1a- and IRE1b-related accessions. Given that M7323S displayed differential basal levels for both IRE1 $a$ and IRE1b, we also included this ecotype in the IRE1b-related category. Overall, IRE1b basal and induced expression remained statistically unchanged in the IRE1a-related accession group except in the reference accession Col-0, which displayed a subtle but significant reduction in the IRE1b mRNA levels (Fig. 2c). By comparing heat-induced IRE1b expression levels in the IRE1a-accession group with Col-0, we also found no statistically significant differences except for a modest increase in the Is-0 ecotype. Subsequently, we tested the basal and heat-induced $I R E 1 b \mathrm{mRNA}$ levels in the IRE1b-accession group. While two ecotypes (MS-0 and Ta-0) exhibited expression patterns consistent with the values reported in the plant eFP browser, we noted that the basal expression levels for M7323S, HR-5, and Fr-2 were different under our experimental conditions (Fig. 2d). Several factors in the experimental set-ups can account for such differences, including the age of plants and light cycle (4 weeks old plants and a 12/12-h light/dark cycle vs. 4 -day-old seedlings and continuous light in our and eFP browser datasets, respectively). After we exposed the IRE1b accession group to heat, we found that the expression of $I R E 1 b$, unlike IRE1 $a$, does not increase after heat stress, with one accession, MS-0, being a notable exception (Fig. $2 \mathrm{~d}$ ). The decreased transcript accumulation in Col-0 is also consistent with the data from the plant eFP browser ${ }^{67}$. When compared to the induced levels of $I R E 1 b$ among 


\begin{tabular}{|l|l|l|l|l|}
\hline Element Sequence & Promoter position & Predicted binding TF & SNP position & GenBank ID \\
\hline acggtataAAAGcgttt & $7616364-7616380$ & DNA binding with one finger (DOF) & $\begin{array}{l}7616379 \\
\text { Bla-5 (G->A) }\end{array}$ & MT344169 \\
\hline aaaATTAttta & $7616531-7616536$ & $\begin{array}{l}\text { Myb-related DNA binding proteins (Golden2, ARR, } \\
\text { Psr) }\end{array}$ & $\begin{array}{l}\text { 7616536 } \\
\text { Bla-5, Dra-1, En-T } \\
\text { (A->T) }\end{array}$ & $\begin{array}{l}\text { MT344169, } \\
\text { MT344170, } \\
\text { MT344171 }\end{array}$ \\
\hline ttAGATccgcc & $7616480-7616490$ & Arabidopsis homeobox protein & $\begin{array}{l}7616481 \\
\text { Dra-1, En-T } \\
(G->A)\end{array}$ & $\begin{array}{l}\text { MT344170, } \\
\text { MT344171 }\end{array}$ \\
\hline
\end{tabular}

Table 2. SNPs found in IRE1a promoter regions across different Arabidopsis accessions that correspond to predicted transcription factor (TF) binding sites. Promoter and SNP positions are based on chromosomal coordinates as listed in TAIR SequenceViewer (https://seqviewer.arabidopsis.org). Nucleotide substitutions in selected accessions are indicated. The promoter sequences newly identified through this study have been submitted to NCBI GenBank under accession numbers listed.

the IRE1b-related ecotypes, we observed a trend of higher heat-induced IRE $1 b \mathrm{mRNA}$ levels in Col- 0 compared to all five accessions with HR-5, Fr-2, and Ta-0 displaying statistically significant differences (Fig. 2d). Analysing fold induction above the basal levels of each accession, the strongest inducers of IRE1b were En-T and MS-0 (Fig. S1d, e). Collectively, we concluded that the IRE1b expression levels were not induced upon treatment with heat in the majority of the tested ecotypes.

Single nucleotide polymorphisms (SNPs) within the IREI $a$ and IRE1b promoter regions. Our results indicate that the transcriptional control of IRE1 $a$ and IRE $1 b$ differs between the two homologues and varies vastly among the natural accessions at both basal and induced levels. To gain more insights into the genetic variation that may be responsible for the observed array of transcriptional dynamics, we sequenced the predicted IRE $1 a$ and IRE $1 b$ promoter regions of Col-0 and other accessions classified into IRE1a- or IRE1b-related groups. We performed multiple sequence alignments of promoter sequences from these selected accessions and the reference sequences of Col- 0 . We detected a number of SNPs in transcription factor binding sites across the IRE1 a promoter regions of Bla-5, Dra-1, and En-T (Table 2, Fig. S2a), which were the top three accessions showing elevated basal IRE1a expression levels. Subsequently, we subjected these polymorphic promoter regions to computational predictions of potential binding sites for transcription factors. Our bioinformatics-aided analysis identified DNA binding with one finger (DOF), Myb-related DNA binding proteins (Golden2, ARR, Psr), and Arabidopsis homeobox protein as the potential regulators (Table 2, Fig. S2a). The three accessions selected for higher basal levels of IRE1a (Bla-5, Dra-1, and En-T) (Table 1) all share the presence of an SNP at position $7616536(\mathrm{~A}->\mathrm{T})$ in the predicted binding site for Myb-related DNA binding proteins, which is unique to this set of promoters and could be one of the factors contributing to elevated basal levels in those ecotypes. All newly identified promoter sequences have been submitted to NCBI GenBank under accession numbers listed in Table 2. No SNPs were detected in the predicted transcription factor binding sites of IRE1b promoter regions, consistent with the previously noted lack of variability in their expression patterns before and after heat treatments.

Transient expression assays to understand IREI $a$ and IRE1 $b$ expression patterns driven through accession-specific promoter sequences. To understand the potential contribution of natural variation in the regulation of IRE1 $a$ and IRE1b expression, we employed transient MUG assays. Moreover, this experiment, at least in part, provided an independent experimental method to support our qRT-PCR-based expression data (Fig. 2). Towards this, we cloned sequence-verified promoter fragments corresponding to IRE1 $a$ and IRE1b from their respective accession groups into a plant Gateway expression vector pAM-PAT-GW-GUS. This led to the generation of transcriptional promoter::GUS reporter fusions designated as pAM-PAT-pIRE1a ${ }^{\mathrm{Col}-0}$-GUS, pAM-PAT-pIRE1a ${ }^{\text {Bla-5}}-$ GUS, pAM-PAT-pIRE1 $a^{\text {Dra-1}}-$ GUS, pAM-PAT-pIRE1 $a^{\text {En-T}}-$ GUS, pAM-PAT-pIRE1 $a^{\text {Is-0 }}$ GUS and pAM-PAT-pIRE1 $\mathrm{a}^{\mathrm{M} 7323 \mathrm{~S}}$-GUS in IRE1-related accession group. Likewise, we generated pAM-PATpIRE $1 b^{\text {Col-0 }}$-GUS, pAM-PAT-pIRE $1 b^{\text {MS-0 }}$-GUS, pAM-PAT-pIRE1b ${ }^{\text {M7323S }}$-GUS, pAM-PAT-pIRE $1 b^{\text {HR-5 }}$-GUS, pAM-PAT-pIRE1b ${ }^{\mathrm{Fr}-2}$-GUS, and pAM-PAT-pIRE $1 \mathrm{~b}^{\mathrm{Ta}-0}$-GUS in the IRE1b-related accession group category. We transiently expressed these two sets of clones in Arabidopsis Col-0 leaves using Agrobacterium-mediated transformation over a three-day period followed by induction by heat stress at $37^{\circ} \mathrm{C}$ for $90 \mathrm{~min}$ (Fig. 3). We collected the leaf tissues, extracted proteins, and quantified activities of $\beta$-glucuronidase (GUS) in each sample via a fluorometric MUG assay ${ }^{71}$. Consistent with our qRT-PCR data, we observed a differential but significant heatmediated induction of GUS activities driven through the set of IRE1a promoters compared to their respective basal levels. It is important to note that the qRT-PCR analyses were performed in the native accession backgrounds, while the MUG assays were done in Col-0 to avoid any accession-specific heterogeneity in gene regulatory mechanisms. Intriguingly, pAM-PAT-pIRE1a ${ }^{\text {Bla-5}}$-GUS displayed an opposite induction trend in Col-0 background (Fig. 3a) compared to its endogenous in planta activity (Fig. 2a). The pIRE1a ${ }^{\text {Bla-5 }}$ sequence contains a unique SNP at nucleotide 7616379 (G->A) (Table 2), which alters a predicted binding site for a DOF transcription factor and might be one of the possible mechanisms explaining the differential inducibility of this promoter

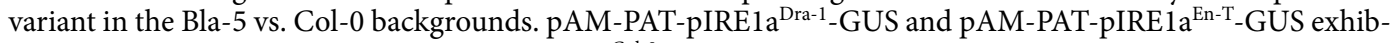
ited comparable induction to pAM-PAT-pIRE1 $\mathrm{a}^{\mathrm{Col}-0}$-GUS, indicating that SNP $7616481(\mathrm{G}->\mathrm{A})$ does not cause elevated basal and/or heat-induced IRE1a promoter activity under the tested conditions. Two pIRE1a constructs 

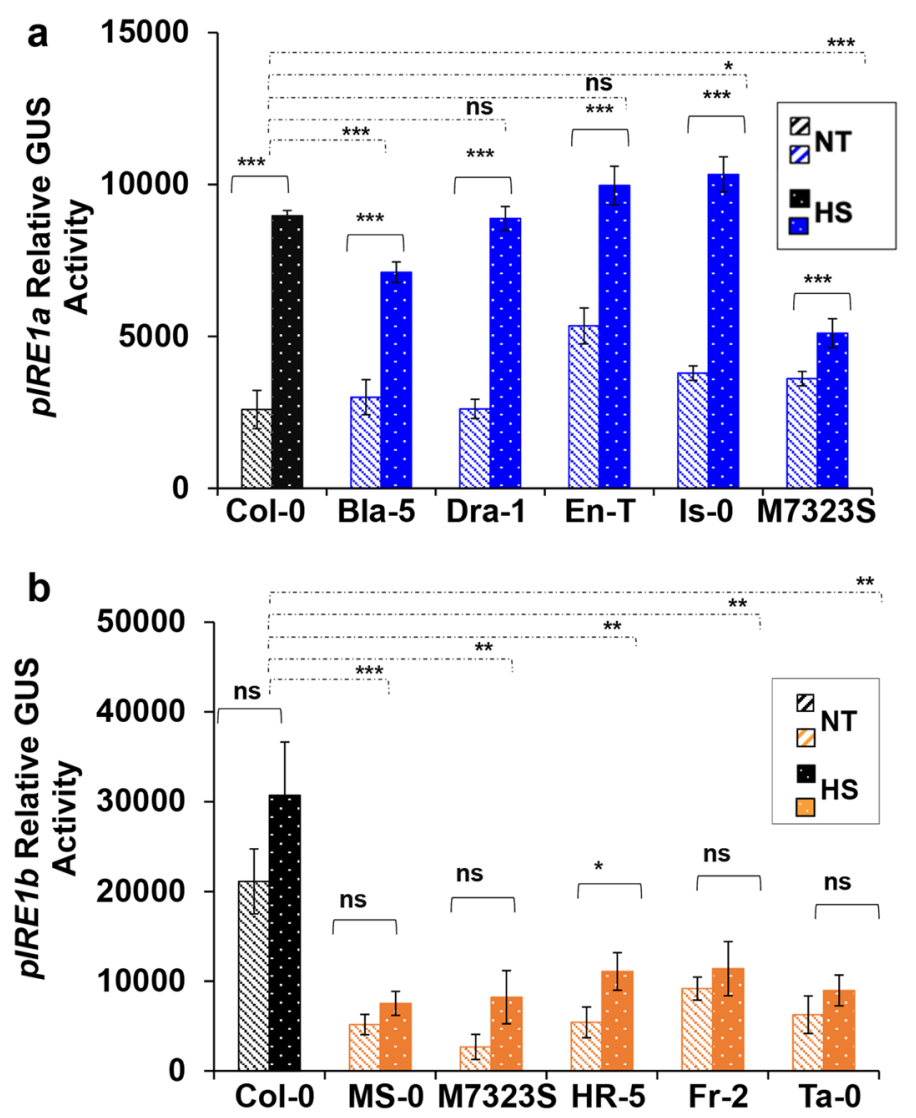

Figure 3. Transient MUG assay to determine basal and heat-induced activities of IRE1a and IRE1b promoters from selected ecotypes. Quantification of ß-glucuronidase (GUS) activity in Arabidopsis Col-0 leaves transiently expressing transcriptional promoter::GUS reporter fusions corresponding to IRE1a (pAM-PAT-pIRE1a ${ }^{\text {Col-0. }}$ GUS, pAM-PAT-pIRE $1 a^{\text {Bla-5}-G U S, ~ p A M-P A T-p I R E 1 ~} a^{\text {Dra-1 }}$-GUS, pAM-PAT-pIRE $1 a^{\text {En-T }}$-GUS, pAM-PATpIRE1 $\mathrm{a}^{\mathrm{Is}-0}$-GUS and pAM-PAT-pIRE1 $\mathrm{a}^{\mathrm{M} 7323 \mathrm{~S}}$-GUS) (a) and IRE1b (pAM-PAT-pIRE1b ${ }^{\mathrm{Col}-0}$-GUS, pAM-PAT-

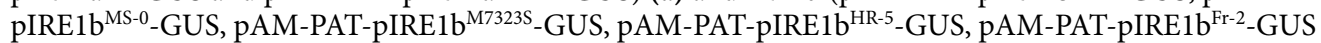
and pAM-PAT-pIRE1b ${ }^{\text {Ta- } 0}$-GUS) (b) before and after heat stress at $37^{\circ} \mathrm{C}$ for $90 \mathrm{~min}$. Promoter activities were determined in extracts of plant tissue via fluorometric MUG assay. Statistical analyses were performed in Excel by One-Way ANOVA. At least three independent biological replicates, each with three technical replicates were performed. Error bars show mean \pm SD. Significant differences are indicated by asterisks $\left({ }^{* *} \mathrm{p}<0.001\right.$, $\left.{ }^{* *} \mathrm{p}<0.01,{ }^{\star} \mathrm{p}<0.05\right)$, while "ns" indicates no statistically significant differences. Solid lines connecting bars represent the comparison of basal to heat-induced expression levels for each individual accession, while dashed lines represent the comparison of induced expression levels between Col- 0 and an indicated accession.

exhibited statistically differential activity levels compared to the pIRE1a ${ }^{\text {Col-0 }}$. While pAM-PAT-pIRE1a ${ }^{\text {Is-0 }}$-GUS was significantly induced in Col-0 background, pAM-PAT-pIRE1a ${ }^{\text {M7323S }}$-GUS showed a reduced activity under the tested conditions (Fig. 3a). Overall, our results suggested that IRE1a promoters derived from the accessions under study exhibit differential levels of activity when tested in the reporter-based transient expression assay in Col-0 background but there is no direct correlation of heat-mediated inducibility of pIRE1a to the SNPs described above under our experimental conditions (Fig. 3a, Table 2).

Similarly to our results with pIRE1a fragments, we observed a trend of increased reporter accumulation for all pIRE $1 b$ constructs upon treatment with heat, although we detected a statistically significant $(p<0.05)$ expression difference only in pAM-PAT-pIRE1b $\mathrm{b}^{\mathrm{HR}-5}$-GUS, corroborating our qRT-PCR results (Fig. 3b). These data also further support a minor role of $I R E 1 b$ induction under heat stress conditions. Subsequently, we compared heatinduced $I R E 1 b$ promoter expression differences between Col-0 and the other five ecotypes. While our qRT-PCR data showed significant $I R E 1 b$ transcript differences upon heat treatment in only three accessions (Fig. 2c,d), the MUG assay highlighted significantly increased IRE $1 b$ promoter::GUS activity in pAM-PAT-pIRE1b ${ }^{\text {Col-0 }}$-GUS compared to the other five tested reporter constructs (Fig. 3b). These results indicate that the data obtained from the highly sensitive MUG assay is largely in agreement with the qRT-PCR results and further delineate the subtle IRE $1 b$ expression differences between Col-0 and other ecotypes. Overall, our results confirm that the heat treatment results in a more dramatic transcriptional response in the IRE1a expression than it is the case for its homologue, IRE1b. A previous study confirmed that IRE1a and IRE1b have distinct expression patterns in Arabidopsis but both can be detected in leaf tissues, biologically validating our experimental design ${ }^{32}$. 
Arabidopsis accessions display differential tolerance to heat and Tm-induced ER stresses. To understand the potential roles of differential IRE1 $a$ and IRE $1 b$ transcript levels in different accessions, we subjected a suite of these 10 ecotypes to heat- and Tm-induced whole-plant ER stress assays. Col- 0 and ire 1a-2 ire $1 b-4$ double mutant plants were used as controls. Specifically, we exposed Arabidopsis seedlings to $42{ }^{\circ} \mathrm{C}$ for $2 \mathrm{~h}$ or liquid MS media supplemented with $0,0.15 \mu \mathrm{g} / \mathrm{mL}$ or $0.3 \mu \mathrm{g} / \mathrm{mL} \mathrm{Tm}$, followed by total weight measurement two (heat) or three (Tm) days later. Overall, we found that all of the accessions displayed a reduction in weight in response to both ER stresses and exhibited generally consistent trends in their levels of sensitivity to ER stress caused by heat and Tm (Fig. 4a,b). In particular, the Bla-5, En-T, Is-0, MS-0, and Fr-2 accessions showed elevated tolerance to one or both types of ER stresses. In contrast, M7323S and Ta-0 were more susceptible to both ER stress-inducing treatments, while Dra-1 was not statistically different than Col-0 (Fig. 4a,b). Moreover, HR-5 displayed a somewhat divergent response, showing significantly enhanced Tm tolerance but a slight increase in heat sensitivity that was not statistically significant. The ire $1 a-2$ ire $1 b-4$ double mutants showed dramatic levels of heat and Tm sensitivity, as previously described ${ }^{46,72}$. Consistent with the elevated basal and heat-induced expression levels of IRE1a in Bla-5 and En-T (Fig. 2a), we showed that these two ecotypes also displayed increased tolerance to ER stress (Fig. 4a,b). The modest improvement in the heat tolerance of Is-0 seedlings might be explained through IRE1-mediated downstream regulatory steps including bZIP60 splicing (see below) rather than its expression per se (Fig. 4c,d). Likewise, MS-0 presented a unique feature, as it is the only accession in the IRE1b-group that was characterised by increased IRE1b expression levels in response to heat (Fig. 2d). On the other hand, the slight increase in ER stress tolerance demonstrated by Fr-2 could also be attributed to IRE1-independent UPR signalling pathways. Finally, the diminished ER tolerance in M7323S and Ta- 0 could potentially be caused by the reduced basal $I R E 1 b \mathrm{mRNA}$ levels in the IRE1b-accession group (Fig. $2 \mathrm{~d}$ and Fig. 4a,b). Overall, we revealed a positive relationship between IRE1a and IRE1b expression levels or their downstream signalling activities, and observed ER stress tolerance in diverse Arabidopsis ecotypes.

Variation in IRE1-mediated bZIP60 processing upon heat stress. A hallmark of the UPR activation in Arabidopsis is the induction of bZIP60 mRNA splicing by IRE1a and IRE1b. Several studies previously documented a marked increase in the $b Z I P 60$ splicing rate upon exposure to heat in Col- 0 33,35,39,58. It is important to note that the plant eFP browser does not include the transcript data corresponding to bZIP60 splice variants. Thus, we experimentally tested the efficacy of $b Z I P 60$ splicing in diverse ecotypes in response to heat. Regardless of the IRE $1 a$ or IRE $1 b$ expression in different ecotypes, we did not observe major differences in the basal bZIP60 splicing efficiency when compared to Col-0. However, we did note a relatively lower $b Z I P 60$ splicing activity in En-T, MS-0, and HR-5 (Fig. 4c,d). Subsequently, we investigated the bZIP60 splicing efficacy under heat-induced conditions. Consistent with our previous study ${ }^{43}$, the reference accession Col-0 showed significant induction of bZIP60 splicing by heat (Fig. 4c,d), whereas the ire1a-2 ire1b-4 double mutant was used as a negative control (Fig. S2b). Except for Bla-5, all the other IRE1a-related ecotypes including Dra-1, En-T, Is-0, and M7323S showed significantly increased $b Z I P 60$ splicing, indicating a successful activation of the IRE1 signalling cascade following heat exposure. Subsequently, we measured differences in heat-induced bZIP60 splicing across different IRE1a-related accessions. We demonstrated that the induced bZIP60 splicing was significantly decreased in Dra-1 and En-T compared to Col-0. On the other hand, Is-0 and M7323S displayed more efficacious bZIP60 splicing compared to Col-0 (Fig. 4c). Finally, we discerned that bZIP60 splicing levels did not fully coincide with both basal and induced levels of IRE1a expression among the accessions within the IRE1a-related group (Fig. 4c). Intriguingly, we observed an inverse relationship between the induced IRE1 $a$ mRNA levels and $b Z I P 60$ splicing, indicating possible existence of compensatory mechanisms between transcriptional and translational activation of IRE1a and its downstream signalling.

For the IRE1b-related accessions (Fig. 4d), we detected high levels of bZIP60 splicing induction in Col-0, MS-0, and M7323S, and a moderate but statistically significant induction in HR-5. While bZIP60 is a bona fide client for both IRE1a and IRE1b during heat stress, we observed some interdependent relationships between IRE1 expression and $b Z I P 60$ splicing efficiency. MS-0 was the only accession in our study that displayed enhanced bZIP60 splicing, which is consistent with the increased levels of IRE1b expression following heat in that accession (Fig. 2d), and could provide a mechanistic explanation of this phenotype. M7323S plants, on the other hand, seemed to rely preferentially on the IRE1a transcriptional induction for bZIP60 splicing, as heat-induced IRE1a levels were significantly induced in that accession. Finally, the HR-5 ecotype was initially selected to be a high basal IRE1b expressor, but turned out to be an under-expressor with a modest induction of $b Z I P 60$ splicing. It is possible that its ability to activate $b Z I P 60$ splicing upon heat could be attributed to the elevated inducibility of IRE1a or other factors that operate at the post-translational level to regulate IRE1a/b protein activity.

Intriguingly, we did not detect any heat-induced bZIP60 splicing in Fr-2 and Ta-0. We first confirmed that our Col-0 specific primers can hybridise to bZIP60 orthologues from Fr- 2 and Ta- 0 by analysing their bZIP60 sequences provided by the 1001 Genomes Project resource ${ }^{25}$; henceforth, we turned to find the answers in the natural history of these two ecotypes. Both accessions originate from Northern and Central Europe (Germany and the Czech Republic), where summers are relatively short and mild, thus prolonged exposure to elevated temperatures $\left(37^{\circ} \mathrm{C}\right)$ might not be common in the natural habitat. Therefore, it is possible that the heat-responsive bZIP60 splicing wasn't shaped by the evolutionary forces in the same way as for several other accessions tested in our experiment. A previous study in mammalian kidney cells demonstrated IRE1's downstream target Xbp1 is spliced at $40^{\circ} \mathrm{C}$ but no splicing was detected at $37^{\circ} \mathrm{C}$ or $43^{\circ} \mathrm{C}$, in contrast to robust induction of Xbp 1 splicing in those cells upon treatments with DTT (inhibitor of disulfide bond formation) and thapsigargin (inhibitor of endoplasmic reticulum $\mathrm{Ca}^{2+}$ ATPase $)^{56}$. These findings suggest that IRE1's downstream splicing is precisely regulated by the temperature, and extreme heat stress may inhibit the ER stress pathway. It is possible that the heat stress of $90 \mathrm{~min}$ at $37^{\circ} \mathrm{C}$ was perceived as acute in the Arabidopsis Fr-2 and Ta- 0 accessions, and resulted in 

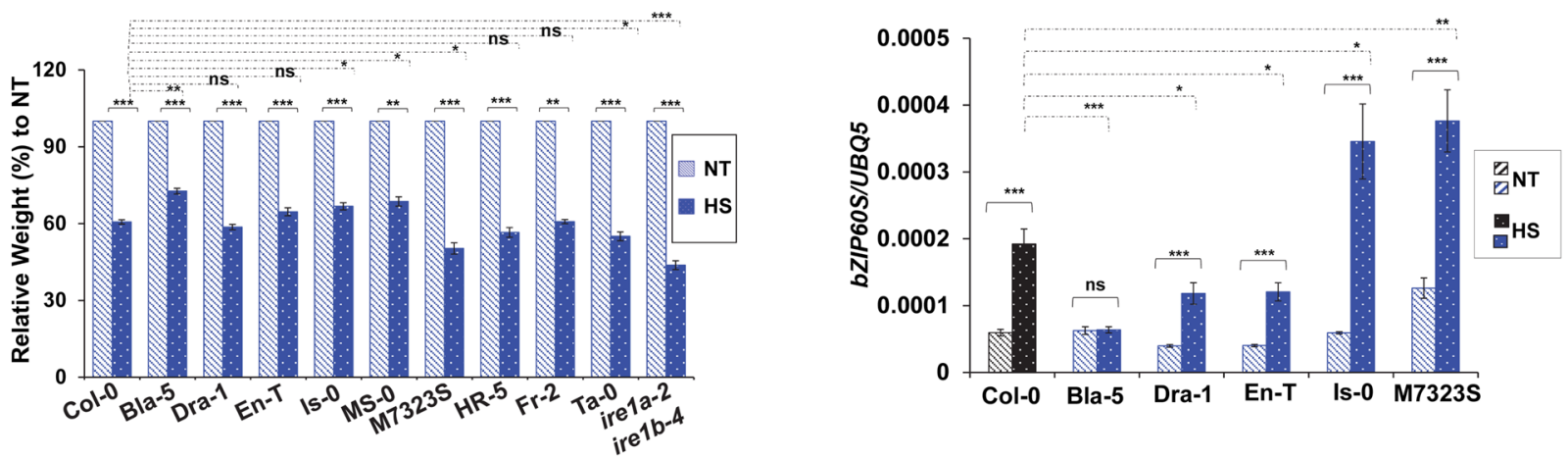

b

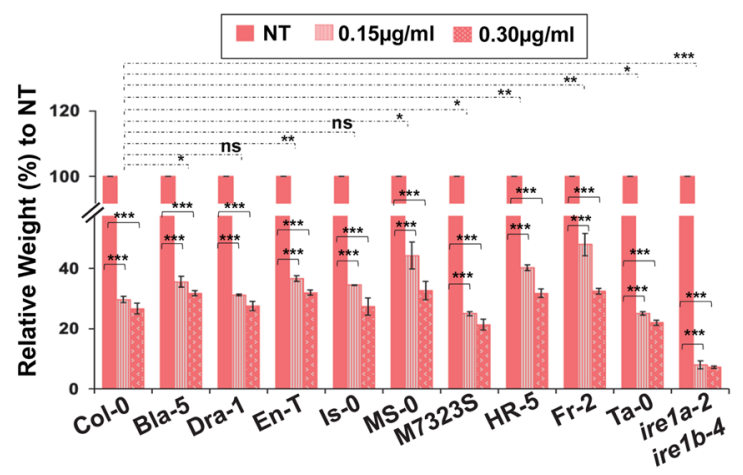

d

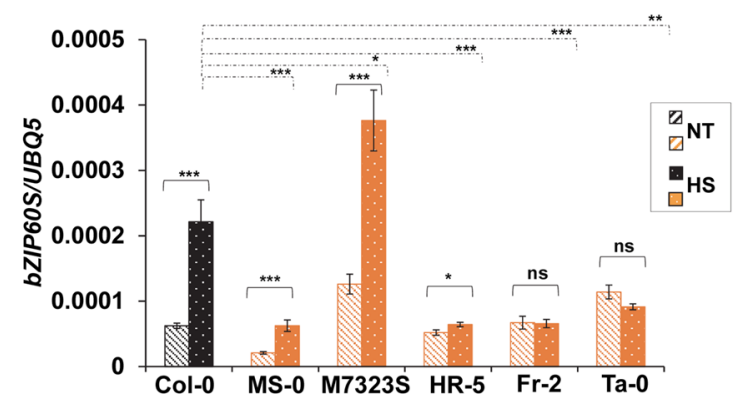

Figure 4. Analysis of ER stress sensitivity and relative heat-induced mRNA expression levels of spliced bZIP60 in selected accessions. (a) Arabidopsis seedlings of indicated ecotypes were grown on solid half-strength MS media for 7 days, and then transferred to liquid half-strength MS media. 9-day-old plants were exposed to $42{ }^{\circ} \mathrm{C}$ for $2 \mathrm{~h}$ or kept at ambient temperature, and a total fresh weight of 10 plants per biological replication was recorded 2 days later. Three biological replications were performed; (b) 7-day-old Arabidopsis seedlings of indicated ecotypes were transferred to liquid half-strength MS media supplemented with the indicated concentration of Tm or mock. The total fresh weight of 10 plants for each of the three biological replications was recorded 3 days following Tm exposure. Statistical analyses for (a) and (b) were performed by One-Way ANOVA. Error bars show mean \pm SD $(n \geq 30)$. Significant differences are indicated by asterisks $\left({ }^{* *} \mathrm{p}<0.001\right.$, $\left.{ }^{* *} \mathrm{p}<0.01,{ }^{*} \mathrm{p}<0.05\right)$, while "ns" indicates no statistically significant differences. Solid lines connecting bars represent the comparison of fresh weight between untreated and treated samples for each individual accession, while dashed lines represent the comparison of fresh weights of stress-treated plants between Col- 0 and an indicated accession. Basal and induced spliced bZIP60 (bZIP60S) expression levels were quantified in selected IRE1a-related accessions (c) or IRE1b-related accessions (d) upon heat stress at $37^{\circ} \mathrm{C}$ for $90 \mathrm{~min}$. Expression levels were measured in leaf tissue of 1-month-old Arabidopsis plants via qRT-PCR and were normalised to housekeeping gene UBQ5 (Ubiquitin 5). Treatment groups are represented according to legends. Dashed bars represent basal expression levels, dotted bars correspond to heat-induced expression levels. Colours indicate accessions grouping (blue-IRE1a-related accessions, orange-IRE1b-related accessions, black-reference accession Col-0). Statistical analyses were performed in Excel by One-Way ANOVA. At least three independent biological replicates, each with three technical replicates were performed. Error bars show mean \pm SD. Significant differences are indicated by asterisks $\left({ }^{* *} \mathrm{p}<0.001,{ }^{* *} \mathrm{p}<0.01,{ }^{\star} \mathrm{p}<0.05\right)$, while "ns" indicates no statistically significant differences. Solid lines connecting bars represent the comparison of basal to heat-induced expression levels for each individual accession, while dashed lines represent the comparison of induced expression levels between Col- 0 and an indicated accession.

an inhibitory, instead of stimulatory, response. However, given that other accessions from the same geographical regions show intact $b Z I P 60$ splicing ability, more work will be needed to ascertain the mechanistic basis for this observation ${ }^{39}$.

Differences in IRE1-mediated UPR signalling in response to biotic stress. Given the central role of SA signalling in inducing UPR in Arabidopsis, we next set out to assess whether SA exerts differential effects on IREI $a$ and IRE1b mRNA levels as well as their downstream UPR signalling activities in our experimental set of Arabidopsis ecotypes. Four-week-old leaves were sprayed with $0.5 \mathrm{mM}$ SA for $6 \mathrm{~h}$, followed by the quantification of basal and SA-induced transcript abundance for IRE1 $a$ and IRE1b in both IRE1a- and IRE1b-group accessions. As expected, IRE1a expression was significantly induced in the reference Col-0, while no change in mRNA levels was detected in the control ire 1a-2 ire 1b-4 double mutant. SA-induced IRE1a transcript was found to be 


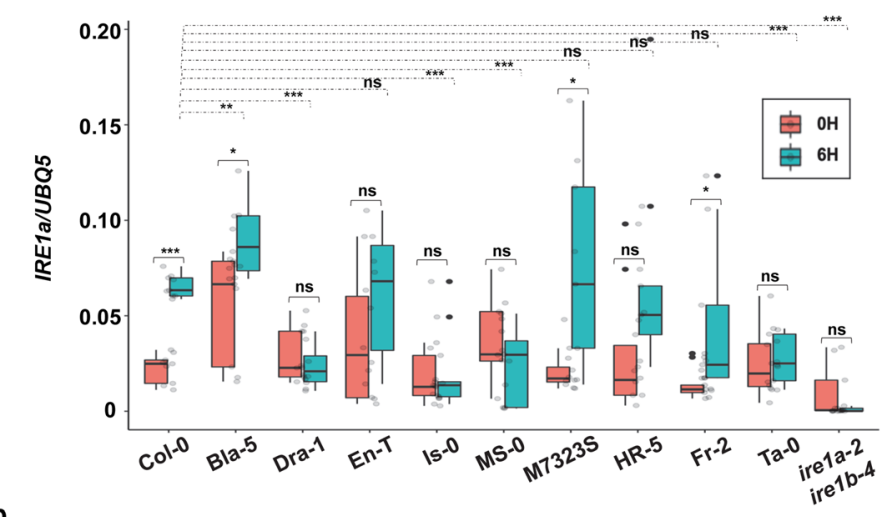

b

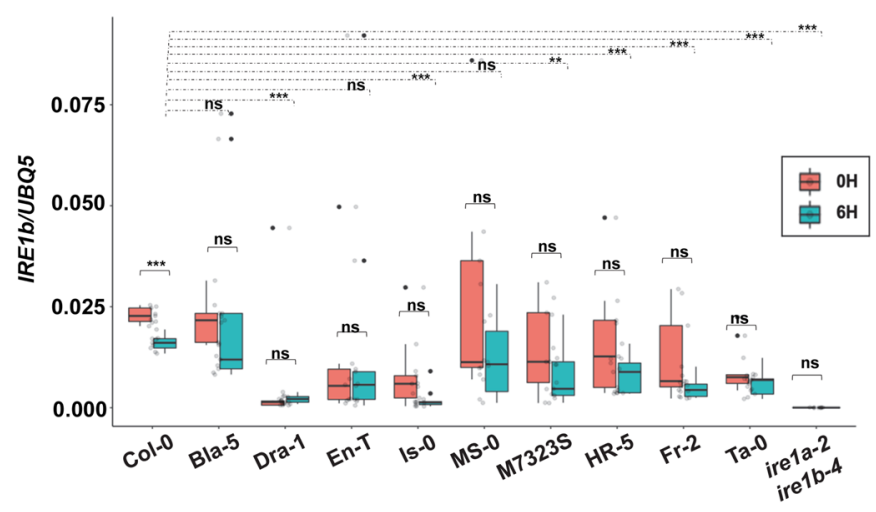

Figure 5. Quantification of relative mRNA levels of IRE1 $a$ and IREIb following Salicylic Acid treatment. Transcript levels of $I R E 1 a$ (a) and $\operatorname{IRE} 1 b$ (b) were quantified using qRT-PCR in leaf tissues of 1-month-old plants that were treated with $0.5 \mathrm{mM} \mathrm{SA}$ or $\mathrm{H}_{2} \mathrm{O}$ (mock) for $6 \mathrm{~h}$. Treatment groups are represented according to legends. All expression levels were normalised to the housekeeping gene UBQ5 (Ubiquitin 5). The box plots extend from the 25th to 75th percentiles and the whiskers extend from the minimum to the maximum levels. Light grey dots represent individual data points. Outliers, shown as dark grey dots, were identified by the test statistics of the geom_boxplot function in ggplot2. Median values were plotted in the boxes with the data generated from three independent biological replicates. Statistical analyses were performed in Excel by oneway ANOVA. Significant differences are indicated by asterisks $\left({ }^{\star * *} p<0.001,{ }^{* *} p<0.01,{ }^{\star} p<0.05\right)$, while "ns" indicates no statistically significant differences. Solid lines connecting bars represent the comparison of basal to SA-induced expression levels for each individual accession, while dashed lines represent the comparison of SA-induced expression levels between Col-0 and an indicated accession.

elevated only in the Bla-5, M7323S, and Fr-2 ecotypes, while no significant change was observed in the remaining members of the IRE1a- and IRE1b-accession groups (Fig. 5a). By comparing the induced levels of IRE1a with those of Col-0, three accessions, namely Bla-5, M7323S, and Fr-2 exhibited significantly higher expression of IRE1 $a$, whereas Dra-1, Is-0 and MS-0 displayed lower, albeit not statistically significant IRE1 $a$ mRNA abundance (Fig. 5a). Interestingly, Dra-1 showed an opposite regulation of IRE1a transcripts when exposed to two diverse ER inducing stressors, heat, and SA, whereas Bla-5 and M7323S consistently displayed the upregulation of IRE1a under both biotic and abiotic ER stress conditions (Fig. 2a,b, and $5 \mathrm{a}$ ). When comparing fold induction above the basal levels of each accession, the strongest inducers of IRE1a were M7323S, Fr-2, Col-0, and HR-5 (Fig. S2c). In agreement with our results indicating the significant reduction of $I R E 1 b$ transcript in response to heat, we also observed a markedly decreased IRE $1 b$ mRNA levels in SA-treated Col-0 plants, while no difference in the transcript abundance was observed in the control ire 1a-2 ire 1b-4 double mutant (Fig. 5b). Similar to the results obtained for heat-induced IRE1 $b$ transcript abundance, no significant change was observed in both IRE1a and IRE1b accession groups (Fig. 5b). By comparing the IRE1b induction levels between Col-0 and other ecotypes, however, we observed an overall reduction of IRE $1 b$ transcript accumulation in our experimental set of accessions (Fig. 5b). When analysing fold induction above the basal levels of each accession, only En-T noticeably induced the IRE $1 b$ transcript, Bla-5 showed a minimal increase, and all the remaining accessions downregulated $I R E 1 b$ expression in response to SA (Fig. S2d). It is worth noting that some of the ecotypes displayed very low levels of basal and/or induced $I R E 1 b$ transcript, potentially masking some expression differences and limiting our ability to reach additional conclusions. Overall, we noted that IRE1b expression does not appear to be substantially changed in the majority of the ecotypes under both biotic and abiotic stress conditions (Fig. 2c,d, and Fig. 5b). 
We next tested bZIP60 splicing in response to SA treatment. Consistent with our heat-induced splicing experiment (Fig. 4c,d), we did not observe any significant differences in basal bZIP60 splicing activity in an independent assay (Fig. 6a), indicating the uniformity in our experimental set-up. Subsequently, we examined the efficacy of SA-induced bZIP60 splicing in the ecotypes under study. The bZIP60 splicing was markedly induced in the leaf tissue of the reference accession Col- 0 treated with $0.5 \mathrm{mM}$ SA for $6 \mathrm{~h}$, while the negative control double mutant ire 1a-2 ire 1b-4 did not exhibit any bZIP60 splicing activity (Fig. 6a). Overall, despite rather low levels of transcript detected in some instances, we observed a significant induction of bZIP60 splicing in all IRE1a- and IRE1b-related ecotypes except the Bla-5 and Ta- 0 accessions. When comparing these results with heat-induced bZIP60 splicing activity, Fr-2 was the only ecotype that exhibits a divergent response pattern to SA. Fr- 2 did not activate bZIP60 splicing under heat stress (Fig. 4d) but was capable of splicing bZIP60 under biotic stress conditions (Fig. 6a), indicating that the ER stress induction in the Fr-2 background is more sensitised towards biotic factors. Moreover, we also observed differences in the amplitude of bZIP60 splicing induction after SA or heat treatments indicating that individual Arabidopsis accessions can differentiate between biotic and abiotic stresses and fine-tune their ER stress responses.

In addition to $b Z I P 60$ splicing, the UPR signalling is also manifested by the production and accumulation of various ER chaperons. Previous studies found a positive relationship of endogenous BiP genes expression and UPR response in Arabidopsis ${ }^{31,34,38,48,73}$. Luminal Binding Protein (BiP) chaperons, also known as HSP70 and GRP78, are very abundant in the ER lumen and thought to bind newly synthesised proteins as they are translocated into the ER, maintain them in a state competent for subsequent folding and oligomerisation, and prevent aggregation of malfolded proteins. $B i P 1, B i P 2$ and $B i P 3$ genes are considered among the most reliable markers for ER stress regulation in plants ${ }^{31,39,42,45,66,71,74}$. Arabidopsis BiP1 and BiP2 are nearly identical in sequence, and the primers used in our analysis detect transcripts of both of those genes. Therefore, we tested the induction of $B i P 1 / 2$ and BiP3 upon SA treatment. Except for Ta-0, all of the tested accessions showed significant upregulation of $B i P 1 / 2$ and $B i P 3$ expression (Fig. $6 \mathrm{~b}$ and $\mathrm{S} 3 \mathrm{a}$ ). While displaying a statistically significant induction $(p<0.05)$, the mRNA of BiP3 in Ta- 0 was accumulated at very low levels. On the other hand, the induction of BiP1/2 expression in this ecotype was not statistically significant. Moreover, we also observed that Col-0 was the strongest inducer of $B i P 3$, while SA-mediated BiP1/2 expression reached its highest levels in three accessions, i.e. Col-0, Bla-5, and En-T; notably, the latter two were among the high basal IRE1a expressors. The double mutant ire 1a-2 ire $1 b-4$ showed a lack of significant BiP1/2 and BiP3 induction (Fig. 6b) ${ }^{31,39,42,45,66,71,74}$.

Endoplasmic reticulum dnaJ domain-containing proteins $3 \mathrm{~A}$ and $3 \mathrm{~B}$ (ERDJ3A and ERDJ3B) are another two molecular co-chaperones that bind to the BiP proteins in mammals and help mediate the protein folding. In Arabidopsis, ERDJ3A is responsible for functional pollen development while ERDJ3B is involved in quality control of ER proteins ${ }^{75}$. Both genes can be used as reliable markers for UPR activity in plants ${ }^{45,73,74}$. ERDJ3A and $E R D J 3 B$ both showed clear patterns of transcriptional induction following SA treatment (Fig. $6 \mathrm{c}$ and S3b) in all accessions with the only exception of M7323S, where ERDJ3A was not significantly induced after SA exposure (Fig. S3b). Similar to our findings for BiP genes expression, the reference accession Col-0 displayed the highest levels of $E R D J 3 A$ and $E R D J 3 B$ induction across all ecotypes tested. The double mutant ire 1a-2 ire 1b-4 showed a lack of significant $E R D J 3 B$ induction but was able to modestly upregulate $E R D J 3 A$, indicating that these two highly related chaperones have distinct transcriptional regulatory mechanisms.

In addition to BiP and ERDJ3 family members, Stromal-Derived Factor 2 (SDF2) represents another important diagnostic ER stress marker ${ }^{76,77}$. SDF2 is a BTH (an SA analogue)-dependent gene ${ }^{78}$ and SDF2 protein can form complexes with ERDJ3B and the BiP proteins to facilitate proper ER homeostasis during PAMP triggered immunity ${ }^{77}$. Consistent with our findings for other ER stress markers, we observed a trend of induction in all Arabidopsis accessions tested (Fig. S3c). Bla-5 and M7323S showed the highest levels of SDF2 induction, along with Col-0 and En-T. Ta- 0 was distinguished by the lowest levels of SDF2 transcript, and ire $1 a-2$ ire $1 b-4$ double mutant showed a lack of significant SDF2 induction. On a general note, as it was the case for the IRE1b expression (Fig. 5b), several ecotypes accumulated very low levels of several ER chaperone transcripts, potentially obscuring additional conclusions about the SA-mediated transcriptional regulation of those genes. Taken together, we noted differential levels of bZIP60 splicing as well as a pronounced induction of downstream UPR chaperons and co-chaperons following SA treatment in the selected natural accessions, indicating that the UPR machinery in different Arabidopsis ecotypes has evolved to cope with versatile surrounding environments.

Response to infection with the bacterial pathogen Pseudomonas syringae. We previously reported that IRE1a and IRE1b are implicated in plant immune responses to a bacterial leaf pathogen, Pseudomonas syringae, including basal defence and establishment of systemic acquired resistance ${ }^{33}$. Through a systematic genetic analysis using a suite of single and double ire 1 mutants, we previously discovered that IRE1a plays a more prominent role in mediating Arabidopsis defences against P. syringae than IRE1b, but both homologues exhibit some degree of functional redundancy and consequently ire 1a ire $1 b$ double mutants display a more profound immune phenotype than the ire $1 a$ single mutants ${ }^{33}$. We hypothesised that the accessions showing higher basal and/or induced levels of IRE1a, IRE1b, SA-mediated bZIP60 splicing, and ER-associated marker genes expression might be better equipped to fight off an infection with virulent $P$. syringae pv. tomato bacteria strain DC3000 (hereafter, Pst DC3000). To test this hypothesis, we subjected Col-0, nine natural accessions, and a hypersusceptible npr1-1 mutant ${ }^{79,80}$ (negative control) to a series of bacterial infection assays ${ }^{81}$. The Pst DC3000 bacteria were pressure-infiltrated into the leaves followed by quantification of the bacterial growth three days later ${ }^{81}$. As expected, the $n p r 1-1$ plants showed a susceptible phenotype with the highest bacterial loads (Fig. 7). Accessions Bla-5, Dra-1, MS-0, M7323S, and Ta-0 showed lower bacterial growth levels compared to Col-0, indicating their relative resistance to Pst DC3000, whereas En-T, Is-0, and HR-5 displayed a similar trend that was not, however, statistically significant. Among all ecotypes tested, Fr-2 was the only one accession that amassed 
a
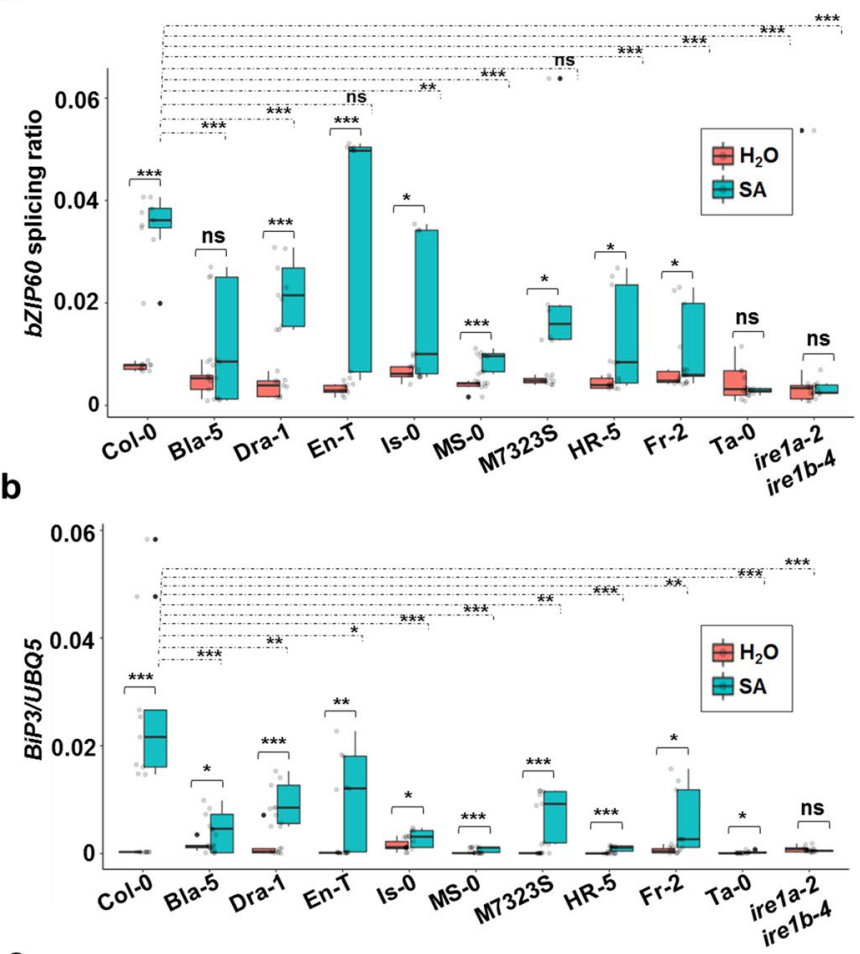

C

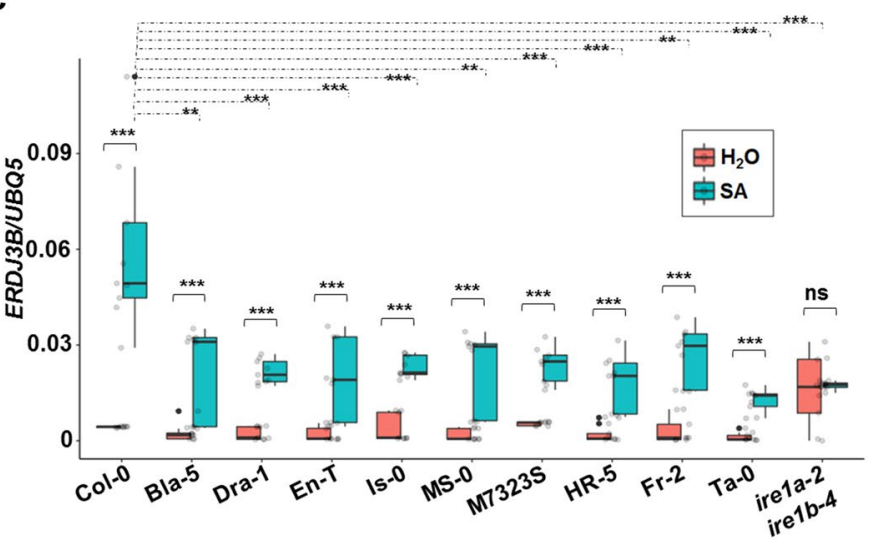

Figure 6. Quantification of $b Z I P 60$ splicing efficacy and relative mRNA levels of ER stress markers BiP3 and ERDJ3B. (a) Transcript accumulation of spliced and unspliced bZIP60 was measured using qRT-PCR in leaf tissues of 1-month-old plants that were treated with $0.5 \mathrm{mM} \mathrm{SA}$ or $\mathrm{H}_{2} \mathrm{O}$ (mock) for $6 \mathrm{~h}$. bZIP60 splicing activity was calculated by normalizing values of spliced bZIP60 to unspliced $b Z I P 60$ transcript abundance. Transcript levels of $B i P 3$ (b) and $E R D J 3 B$ (c) were quantified using qRT-PCR in leaf tissues of 1-month-old plants that were treated with $0.5 \mathrm{mM} \mathrm{SA}$ or $\mathrm{H}_{2} \mathrm{O}$ (mock) for $6 \mathrm{~h}$. Treatment groups are represented according to legends. All expression levels shown in panels a-c were measured in leaf tissues of 1-month-old Arabidopsis plants via qRT-PCR and were normalised to the housekeeping gene UBQ5 (Ubiquitin 5). The box plots extend from the 25th to 75th percentiles and the whiskers extend from the minimum to the maximum levels. Light grey dots represent individual data points. Outliers, shown as dark grey dots, were identified by the test statistics of the geom_boxplot function in ggplot2. Median values were plotted in the boxes with the data generated from three independent biological replicates. Statistical analyses were performed in Excel by one-way ANOVA. Significant differences are indicated by asterisks $\left({ }^{* *} \mathrm{p}<0.001,{ }^{* \star} \mathrm{p}<0.01,{ }^{\star} \mathrm{p}<0.05\right)$, while "ns" indicates no statistically significant differences. Solid lines connecting bars represent the comparison of basal to SA-induced expression levels for each individual accession, while dashed lines represent the comparison of SA-induced expression levels between Col-0 and an indicated accession.

slightly higher, although not statistically significant, pathogen loads when compared to Col-0 (Fig. 7). Double mutant ire1a-2 ire 1b-4 displayed significant susceptibility compared to Col-0, as reported previously ${ }^{33}$ (Fig. S4). 


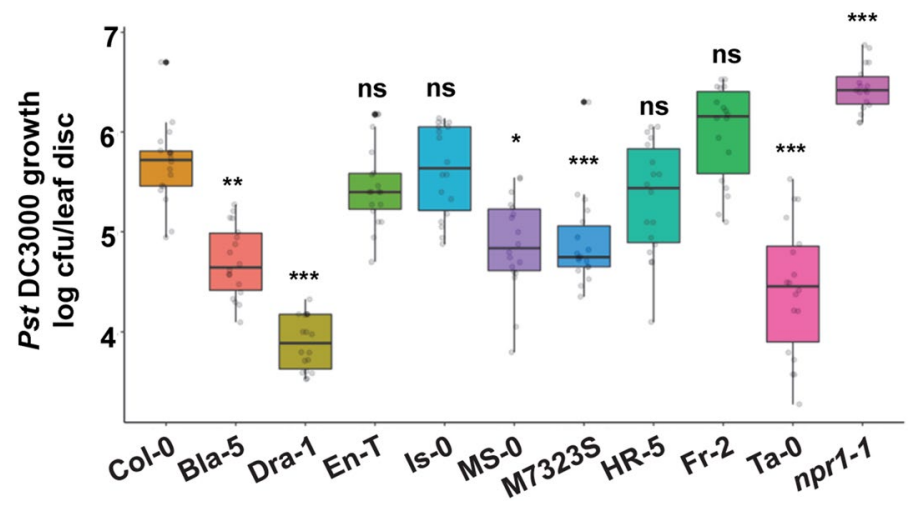

Figure 7. Natural variation of resistance to Pseudomonas syringae pv. tomato DC3000 among selected Arabidopsis accessions. Leaves of 4 weeks old plants were syringe infiltrated with Pseudomonas syringae pv. tomato strain DC3000 (Pst DC3000). In planta bacterial growth was quantified at 3 days postinoculation. The box plots extend from 25th to 75th percentiles and whiskers extend from the minimum to the maximum levels. Light grey dots represent individual data points. Outliers, shown as dark grey dots, were identified by the test statistics of the geom_boxplot function in ggplot2. Median values were plotted in the boxes with the data generated from three independent biological replicates. Statistical analyses were performed in Excel by One-Way ANOVA. Significant differences are indicated by asterisks ${ }^{* * *} p<0.001,{ }^{* *} p<0.01$, $\left.{ }^{\star} \mathrm{p}<0.05\right)$, while "ns" indicates no statistically significant differences. Black asterisks are representing the comparison of resistance or susceptibility of respective accession compared to Col-0.

While the plant immune response is a complex process that engages numerous signalling pathways, we detected some parallels between pathogen resistance and expression of IRE1 genes and downstream ER stress markers. Our initial hypothesis has proven correct for several accessions. For example, Dra-1, the accession with the highest basal IRE1 a levels, showed increased heat-induced IRE1a expression, increased bZIP60 splicing after the heat and SA treatments, and strong SA-mediated inducibility of BiP1/2, BiP3, ERDJ3A, ERDJ3B, and SDF2. Dra-5 was also the most resistant accession in our study and accumulated bacterial loads $\sim 100$ times lower than those of Col-0. Another resistant ecotype HR-5 (high basal IRE1b accession) showed reduced sensitivity to Tm, high levels of $b Z I P 60$ splicing after the heat and SA treatments, and strong SA-mediated inducibility of $B i P 1 / 2, B i P 3, E R D J 3 A, E R D J 3 B$, and SDF2. Analogous conclusions can be drawn for Bla-5 (high basal IRE1a accession), which displayed a similar data trend with the exception of the bZIP60 splicing, which was not significantly induced. Interestingly, MS-0 and M7323S are two accessions that were selected as low IRE1a and/ or IRE1b expressors, yet displayed increased bZIP60 splicing after the heat and SA treatments and substantial SA-mediated inducibility of almost all tested ER markers. This increased induction of bZIP60 splicing and ER markers are consistent with enhanced disease resistance phenotypes of MS- 0 and M7323S. On the other hand, $\mathrm{Ta}-0$ is an accession that we initially selected based on reduced basal IRE1b transcript levels. Predictably, in our expression studies, we observed a complete lack of bZIP60 inducibility and very low overall levels of the ER markers expression, as well as increased sensitivity to both heat and Tm. Yet, despite its poor ER-associated transcriptional signature, Ta-0 showed surprisingly low levels of Pst DC3000 growth, ranking as the second most resistant accession in our analysis. This finding points towards the likely existence of compensatory mechanisms, where other defence-related pathways might have been hyperactive to fend off the pathogens in their local environment while the induction of UPR machinery is impaired. In conclusion, our phytopathology analyses provided several lines of evidence for an interrelation between the relative fitness of the ER signalling pathways and overall immunity to Pst DC3000 infection.

Euclidean clustering analysis reveals integrative transcriptional and phenotypic patterns. To integrate our findings and uncover novel patterns and relationships between the ecotypes, we next performed Euclidean clustering analysis of the transcriptional and phenotypic responses in our panel of accessions (Fig. 8). Is-0, Fr- 2 and Col-0 ecotypes share common geographical origins: Is-0 originates from Isenberg, Germany, Fr-2 stems from the neighbouring city of Frankfurt, Germany, and Col-0, often incorrectly attributed to Columbia, Missouri, USA, actually originates from north-western Poland ${ }^{22}$. These three accessions clustered together in our analysis, demonstrating predominantly consistent trends of stress-induced transcriptional responses, and similar levels of susceptibility to bacterial disease. MS-0 of Moscow, Russia, and Ta-0, hailing from Tabor, Czech Republic, both low basal expressors of IRE1b, clustered in a separate group distinguished by low amplitudes of transcriptional ER stress responses but high levels of bacterial resistance, indicating that Arabidopsis immunity to Pst DC3000 can be variably tied to UPR signalling depending on the specific genetic background. It is worth noting that MS-0 showed a positive relationship between high SDF2 transcript levels and enhanced tolerance to heat and $\mathrm{Tm}$, which distinguished it as the only ecotype with such phenotypic features. Interestingly, our analysis also uncovered an interrelation between the efficacy of bZIP60 splicing and expression of BiP3 (Fig. 6), which is consistent with the notion that $B i P 3$ itself has been shown to be one of the main transcriptional targets of active bZIP60 transcription factor ${ }^{82}$. This relationship is further reinforced by a positive feedback loop as an 


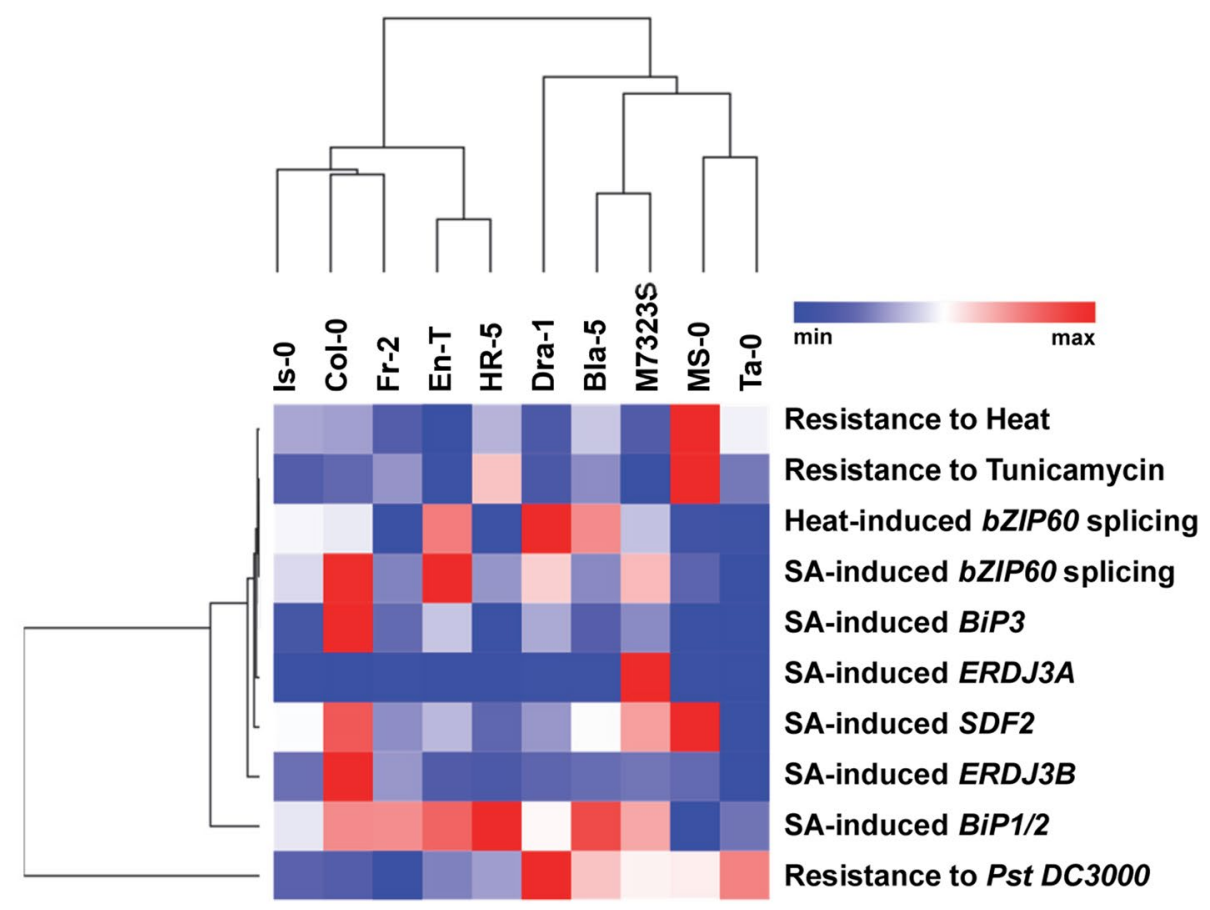

Figure 8. Heat map comparisons of differential gene expression, whole-plant ER stress sensitivity, and pathology phenotypes of selected Arabidopsis accessions. Responses of the selected accessions to different treatments were integrated into a heat map. Euclidean distance was used as a metric for KMeans and hierarchical clustering. In hierarchical clustering, the average was the linkage method. Accession names and treatments are indicated. Colours from red to blue indicate high to low expression/trait intensity.

active bZIP60 is also able to activate its own expression through an ERSE (ER response element)-like element present in its promoter ${ }^{48}$. Expression trends of $B i P 1 / 2$ were independent of other markers, which is not unexpected since BiP1 and BiP2 are strongly and ubiquitously expressed, and weakly regulated by bZIP60 ${ }^{31}$. ERDJ3A, which has been previously shown to be a heat- and bZIP60-independent ER stress marker ${ }^{83}$, showed expression patterns that clustered together with multiple ER chaperones following the immune stressor SA treatment. This observation is consistent with the presence of SA-inducible heat shock-like translocon1 (TL1) cis-regulatory elements in ERDJ3A promoter ${ }^{71,83}$ and indicates that additional transcriptional regulators, such as $\mathrm{TBF} 1^{71}$, might operate to bridge the UPR signalling with SA-mediated immune responses. In support of this hypothesis, TBF1 was previously shown to regulate SA-induced expression of BiP2 via TL1 motifs ${ }^{11}$. Given the complexity of the plant immune response, it is predictable that the levels of bacterial resistance don't show an absolute concurrence with any specific ER stress marker(s) in our analysis; however, these results prove valuable to provide interesting insights into the ecological and evolutionary relationship between the UPR and immunity to Pst DC3000.

\section{Conclusion}

The genetic variation found in populations from different natural environments demonstrates the extent of local adaptation and helps gain insights into the molecular underpinnings of plant adaptive responses. This study characterised the ER stress responses in a panel of 10 natural Arabidopsis accessions and uncovered a number of variations in their UPR signatures following exposure to biotic and abiotic stress factors. Our work suggests that both Arabidopsis IRE1 homologues, as well as their downstream signalling components, are subject to variation imposed by the evolutionary forces both at the genomic and gene regulation levels. We provided new insights into the natural diversity of a ubiquitous and evolutionarily conserved cellular stress signalling pathway, and our discoveries can form a foundation to engineer climate-resilient crop plants; a necessity for a sustainable future.

\section{Materials and methods}

Plant material and growth conditions. The seeds of selected A. thaliana accessions listed in Table 1 were ordered from the Arabidopsis Biological Resource Center (ABRC, Ohio State University, Columbus, OH, USA). All the seeds were sown on sterilised soil (SunGro Horticulture, Super-Fine Germinating Mix) in individual pots. The seeds were stratified for seven days in a cold room facility at $4{ }^{\circ} \mathrm{C}$. The pots were then transferred to a controlled growth room facility $\left(12 \mathrm{~h}\right.$ light $/ 12 \mathrm{~h}$ dark photoperiod; $21^{\circ} \mathrm{C} ; 100 \mu \mathrm{mol} / \mathrm{m}^{2} / \mathrm{s} \mathrm{light}$ intensity and $40 \%$ relative humidity). 10-15 days old seedlings were then transplanted into 72 -well flats for growth ( 1 month) and subsequent experimentation. 
Plant photography. The pictures of the plants representing individual ecotypes were taken by NIKON D5600 camera and were edited using Adobe Photoshop (Version: 21.2.4).

Selection of accessions. The accessions (Table 1) were selected based on IRE1a and IRE1b expression patterns in Arabidopsis eFP (electronic Fluorescent Pictograph) browser, available at https://www.bar.utoronto. $\mathrm{ca} /$. Logarithmic fold-change values were provided by ePLANT, with relative logarithmic values above 0.7 and below -0.8. The accessions that showed the highest extent of variation, characterised by the highest and lowest expression values for IRE $1 a$ and IRE $1 b$ were selected.

Heat stress assays and salicylic acid treatments. 1-month-old Arabidopsis plants grown in soil were exposed to heat stress at $37^{\circ} \mathrm{C}$ for $90 \mathrm{~min}$ in an incubator, then leaf tissue was sampled. For phytohormone treatment, aerial parts of 1-month-old soil-grown Arabidopsis plants were sprayed with $0.5 \mathrm{mM} \mathrm{SA}$, covered with a dome for $2 \mathrm{~h}$, and leaf samples were collected after $6 \mathrm{~h}$ post-treatment. At least three leaves derived from three independent plants were collected for each ecotype/treatment/time point combination.

ER stress assays. Seeds from different Arabidopsis accessions were washed with $70 \%$ Ethanol and $0.05 \%$ Triton and then stratified at $4{ }^{\circ} \mathrm{C}$ for 3 days on half-strength solid Murashige Skoog (MS) media plates (Phytotechnology Labs, Overland Park, KS, USA). After stratification, MS plates were transferred to growth chambers ( $12 \mathrm{~h}$ light $/ 12 \mathrm{~h}$ dark photoperiod; $21^{\circ} \mathrm{C} ; 100 \mu \mathrm{mol} / \mathrm{m}^{2} / \mathrm{s}$ light intensity and $40 \%$ relative humidity). $0.15 \mu \mathrm{g} / \mathrm{mL}$ or $0.30 \mu \mathrm{g} / \mathrm{mL}$ of Tunicamycin (Tm) (Tocris Bioscience; 3516/10) were used as a chemical ER stressor. 7 days old seedlings were transferred to liquid half-strength MS media with or without the appropriate concentration of $\mathrm{Tm}$. The total fresh weight of 10 plants for each biological replications was recorded 3 days following $\mathrm{Tm}$ exposure. For heat stress, Arabidopsis seedlings were grown on solid half-strength MS media for 7 days and then transferred to liquid half-strength MS media. 9 days old Arabidopsis plants were exposed to $42{ }^{\circ} \mathrm{C}$ for $2 \mathrm{~h}$ and the total weight was recorded 2 days later.

mRNA quantification and bZIP60 splicing. Gene expression analysis was conducted as described previously $^{84}$. In brief, leaf tissue from 1-month-old plants was collected at designated time points. At least three leaves derived from three independent plants were collected for each genotype/ treatment/ time point combination. Trizol reagent (Invitrogen) was used to extract total RNA and DNase I (Ambion) was applied to remove DNA contaminants. $10 \mu \mathrm{g}$ of total RNA were reverse transcribed using SuperScript III first-strand RT-PCR kit (Invitrogen), and quantitative gene expression analysis was determined using GoTaq qPCR Master Mix (Promega) with transcript-specific primers in a RealPlex S MasterCycler (Eppendorf). The Ct values were normalised to ubiquitin 5 (UBQ5) gene. bZIP60 splicing assays were performed as described in Moreno et al. ${ }^{33}$. Briefly, we used a common forward primer and a pair of reverse primers that specifically hybridise to either the unspliced or spliced variants of cDNA originated from bZIP60 mRNA, respectively. This allows for the detection of two specific qRT-PCR products corresponding to unspliced and spliced bZIP60 variants (Fig. S5). Primers used for qRT-PCR are listed in Table S1.

Preparation of promoter::GUS constructs. DNA extraction was performed from 1 month old plants with $200 \mu \mathrm{l}$ CTAB extraction buffer (2\% cetyl-trimethyl ammonium bromide, $100 \mathrm{mM}$ tris [pH 8.0], $20 \mathrm{mM}$ EDTA pH [8.0], 1.4 M NaCl, 0.5\% $\beta$-Mercaptoethanol, 2\% polyvinyl pyrrolidone). The promoter region of IRE1a $(\sim 1.267 \mathrm{~kb})$ and $\operatorname{IRE} 1 b(\sim 1.477 \mathrm{~kb})$ from different accessions were amplified from genomic DNA by PCR using Phusion Polymerase (Thermo Scientific) with attB-flanked primers (Table S1). The PCR products were cloned into pDONR207 Gateway vector via BP reactions (Invitrogen). After confirming the entry clones through PCR and Sanger sequencing (primers listed in Table S1), destination clones were constructed by LR reactions with binary Gateway vector pAM-PAT-GW-GUS and confirmed through PCR and Sanger sequencing. The plant expression vector pAM-PAT-35S-GW-GUS was a gift from Drs. Nico Dissmeyer and Imre Somssich (Addgene plasmid \# 80,678; https://n2t.net/addgene:80678; RRID:Addgene_80678). The resulting pAM-PAT-promoterGUS constructs were transformed into Agrobacterium tumefaciens (strain GV3101) for transient expression assays.

IRE1 $a$ and IRE1b promoter analyses. We analysed the obtained promoter sequences using 4Peaks software (https://nucleobytes.com/4peaks/index.html). The confirmed sequences were aligned with the IRE1a or IRE $1 b$ promoter sequence of the reference accession Col-0 using MultAlin website (https://multalin.toulouse. inra.fr/multalin/) (Fig. S2). The promoter sequences from Col-0 were used to predict putative transcription factor binding sites using the software MatInspector (https://www.genomatix.de/online_help/help_matinspect or/matinspector_help.html) and the website PlantRegMap (https://plantregmap.gao-lab.org). The predicted TF target sequences were matched with the SNPs identified from MultAlin website. The newly identified TF binding sites with SNPs have been submitted to NCBI GenBank under the following accession numbers: MT344169, MT344170, and MT344171.

Quantitative GUS assay. 1-month-old Col-0 plants were agroinfiltrated with a needleless syringe as described previously ${ }^{85}$. Three days post-inoculation, the plants were exposed to heat stress in an incubator at $37^{\circ} \mathrm{C}$ for $90 \mathrm{~min}$. Immediately following the heat stress, the tissues were collected and ground under liquid nitrogen. Total proteins from the harvested tissue were extracted with extraction buffer $\left(50 \mathrm{mM} \mathrm{NaPO}_{4}[\mathrm{pH}\right.$ 7.0], $1 \mathrm{mM} \mathrm{Na}{ }_{2}$ EDTA, $0.1 \%$ SDS, $0.1 \%$ Triton X-100, protease inhibitor for plant extracts [Sigma], and $10 \mathrm{mM}$ 
$\beta$-mercapethanol) as described previously ${ }^{71}$. Followed by centrifugation $\left(10 \mathrm{~min}, 4000 \times \mathrm{g}, 4^{\circ} \mathrm{C}\right)$ the supernatants were collected, and protein concentration was quantified using Bradford Reagent (Sigma). The extracted proteins were incubated with $1 \mathrm{mM}$ MUG (4-methylumbelliferyl $\beta$-D-glucuronide) to quantify GUS activity. $1 \mathrm{M} \mathrm{Na}_{2} \mathrm{CO}_{3}$ was used as a stop buffer to terminate the reaction and fluorescence was measured with a microplate reader (Tecan) with an excitation wavelength of $365 \mathrm{~nm}$, an emission wavelength of $455 \mathrm{~nm}$ and a filter wavelength of $430 \mathrm{~nm}$. The relative MUG values were obtained by normalizing data to the Bradford assay. The experimental procedures were adjusted based on a previously published protocol ${ }^{86}$.

Bacterial strains and bacterial growth quantification. Pseudomonas syringae pv. tomato DC3000 (Pst DC3000) was used for pathogen infection and quantification assay. 1-month-old soil-grown plants were syringe-infiltrated with Pst DC3000 $\left(\mathrm{OD}_{600}=0.0002\right)$. 3 leaves/plant, 6 plants/replication, and at least three independent biological replications were performed. Bacterial growth was quantified three days of post inoculation as described previously ${ }^{81}$.

Heat map and Euclidean clustering analysis. The heat map was generated using the website Morpheus (https://software.broadinstitute.org/morpheus/). Euclidean distance was used as a metric for KMeans and Hierarchical clustering. In hierarchical clustering, the average was the linkage method. Colors from red to blue indicate high to low expression/trait intensity.

Statistical analysis. Statistical differences were calculated by one-way ANOVA in Excel and R. ggplot2 was used to make graphs shown in Figs. 5, 6, and 7, and supplemental Figs. 3 and 4. Statistically significant differences are indicated with ${ }^{\star} \mathrm{p}<0.05,{ }^{* *} \mathrm{p}<0.01,{ }^{* * *} \mathrm{p}<0.001$, and ${ }^{\star * * *} \mathrm{p}<0.0001$.

Creative commons. Natural Variation of IRE1 in Arabidopsis" by Taiaba Afrin is licensed under CC BY-SA 4.0. To view a copy of this license, visit https://creativecommons.org/licenses/by-sa/4.0.

\section{Data and materials availability}

All data needed to evaluate the conclusions in this article are present in the paper and/or the Supplementary Materials. Additional data related to this paper may be requested from the authors. The reported mutant seeds and plasmids can be provided by KPM pending scientific review and a completed material transfer agreement.

Received: 30 May 2020; Accepted: 19 October 2020

Published online: 05 November 2020

\section{References}

1. Claeys, H. \& Inze, D. The agony of choice: how plants balance growth and survival under water-limiting conditions. Plant Physiol. 162, 1768-1779. https://doi.org/10.1104/pp.113.220921 (2013).

2. Aitken, S. N., Yeaman, S., Holliday, J. A., Wang, T. \& Curtis-McLane, S. Adaptation, migration or extirpation: climate change outcomes for tree populations. Evol. Appl. 1, 95-111. https://doi.org/10.1111/j.1752-4571.2007.00013.x (2008).

3. Jump, A. S. \& Penuelas, J. Running to stand still: adaptation and the response of plants to rapid climate change. Ecol. Lett. 8, $1010-1020$ (2005)

4. Fournier-Level, A. et al. A map of local adaptation in Arabidopsis thaliana. Science 334, 86-89. https://doi.org/10.1126/scien ce.1209271 (2011)

5. Alonso-Blanco, C. \& Koornneef, M. Naturally occurring variation in Arabidopsis: an underexploited resource for plant genetics. Trends Plant Sci. 5, 22-29. https://doi.org/10.1016/s1360-1385(99)01510-1 (2000).

6. Bouchabke, O. et al. Natural variation in Arabidopsis thaliana as a tool for highlighting differential drought responses. PLoS ONE 3, e1705. https://doi.org/10.1371/journal.pone.0001705 (2008).

7. Shindo, C., Bernasconi, G. \& Hardtke, C. S. Natural genetic variation in Arabidopsis: tools, traits and prospects for evolutionary ecology. Ann. Bot. 99, 1043-1054. https://doi.org/10.1093/aob/mcl281 (2007).

8. Koornneef, M., Alonso-Blanco, C. \& Vreugdenhil, D. Naturally occurring genetic variation in Arabidopsis thaliana. Annu. Rev. Plant Biol. 55, 141-172. https://doi.org/10.1146/annurev.arplant.55.031903.141605 (2004).

9. Horton, M. W. et al. Genome-wide patterns of genetic variation in worldwide Arabidopsis thaliana accessions from the RegMap panel. Nat. Genet. 44, 212-216. https://doi.org/10.1038/ng.1042 (2012).

10. Hoffmann, M. H. Biogeography of Arabidopsis thaliana (1.) heynh. (Brassicaceae). J. Biogeogr. 29, 125-134 (2002).

11. Katori, T. et al. Dissecting the genetic control of natural variation in salt tolerance of Arabidopsis thaliana accessions. J. Exp. Bot. 61, 1125-1138. https://doi.org/10.1093/jxb/erp376 (2010).

12. Pérez-Pérez, J. M., Serrano-Cartagena, J. \& Micol, J. L. Genetic analysis of natural variations in the architecture of Arabidopsis thaliana vegetative leaves. Genetics 162, 893-915 (2002).

13. Pigliucci, M. \& Schlichting, C. D. Reaction norms of Arabidopsis (Brassicaceae). III. Response to nutrients in 26 populations from a worldwide collection. Am. J. Bot. 82, 1117-1125 (1995).

14. Koornneef, M., Alonso-Blanco, C., Peeters, A. J. \& Soppe, W. Genetic control of flowering time in Arabidopsis. Annu. Rev. Plant Physiol. Plant Mol. Biol. 49, 345-370. https://doi.org/10.1146/annurev.arplant.49.1.345 (1998).

15. Ungerer, M. C., Halldorsdottir, S. S., Modliszewski, J. L., Mackay, T. F. \& Purugganan, M. D. Quantitative trait loci for inflorescence development in Arabidopsis thaliana. Genetics 160, 1133-1151 (2002).

16. Alonso-Blanco, C., Bentsink, L., Hanhart, C. J., Blankestijn-de Vries, H. \& Koornneef, M. Analysis of natural allelic variation at seed dormancy loci of Arabidopsis thaliana. Genetics 164, 711-729 (2003).

17. Langridge, J. \& Griffing, B. A study of high temperature lesions in Arabidopsis thaliana. Aust. J. Biol. Sci. 12, 117-135 (1959).

18. 18Thomashow, M. Arabidopsis thaliana as a model for studying mechanisms of plant cold tolerance. Arabidopsis, 807-834 (1994).

19. Adam, L. et al. Comparison of Erysiphe cichoracearum and E. cruciferarum and a survey of 360 Arabidopsis thaliana accessions for resistance to these two powdery mildew pathogens. Mol. Plant Microbe Interact. 12, 1031-1043. https://doi.org/10.1094/ MPMI.1999.12.12.1031 (1999). 
20. Weinig, C., Stinchcombe, J. R. \& Schmitt, J. QTL architecture of resistance and tolerance traits in Arabidopsis thaliana in natural environments. Mol. Ecol 12, 1153-1163. https://doi.org/10.1046/j.1365-294x.2003.01787.x (2003).

21. Swarup, K. et al. Natural allelic variation identifies new genes in the Arabidopsis circadian system. Plant J. 20, 67-77. https://doi. org/10.1046/j.1365-313x.1999.00577.x (1999).

22. Koornneef, M. \& Meinke, D. The development of Arabidopsis as a model plant. Plant J. 61, 909-921 (2010).

23. Mitchell-Olds, T. Arabidopsis thaliana and its wild relatives: a model system for ecology and evolution. Trends Ecol. Evol. 16, 693-700 (2001).

24. Arabidopsis Genome, I. Analysis of the genome sequence of the flowering plant Arabidopsis thaliana. Nature 408, 796-815. https ://doi.org/10.1038/35048692 (2000).

25. 25 Genomes Consortium. Electronic address, m. n. g. o. a. a. \& Genomes, C. 1,135 Genomes reveal the global pattern of polymorphism in Arabidopsis thaliana. Cell 166, 481-491, http://doi.org/https://doi.org/10.1016/j.cell.2016.05.063 (2016).

26. Michalak, M., Corbett, E. F., Mesaeli, N., Nakamura, K. \& Opas, M. Calreticulin: one protein, one gene, many functions. Biochem. J. 344(Pt 2), 281-292 (1999).

27. Latham, K. E. Endoplasmic reticulum stress signaling in mammalian oocytes and embryos: life in balance. Int. Rev. Cell. Mol. Biol. 316, 227-265. https://doi.org/10.1016/bs.ircmb.2015.01.005 (2015).

28. Wu, H., Ng, B. S. \& Thibault, G. Endoplasmic reticulum stress response in yeast and humans. Biosci. Rep. https://doi.org/10.1042/ BSR20140058 (2014).

29. Afrin, T., Diwan, D., Sahawneh, K. \& Pajerowska-Mukhtar, K. Multilevel regulation of endoplasmic reticulum stress responses in plants: where old roads and new paths meet. J. Exp. Bot. 71, 1659-1667. https://doi.org/10.1093/jxb/erz487 (2020).

30. Korner, C. J., Du, X., Vollmer, M. E. \& Pajerowska-Mukhtar, K. M. Endoplasmic reticulum stress signaling in plant immunity-at the crossroad of life and death. Int. J. Mol. Sci. 16, 26582-26598. https://doi.org/10.3390/ijms161125964 (2015).

31. Iwata, Y., Fedoroff, N. V. \& Koizumi, N. Arabidopsis bZIP60 is a proteolysis-activated transcription factor involved in the endoplasmic reticulum stress response. Plant Cell 20, 3107-3121. https://doi.org/10.1105/tpc.108.061002 (2008).

32. Koizumi, N. et al. Molecular characterization of two Arabidopsis Irel homologs, endoplasmic reticulum-located transmembrane protein kinases. Plant Physiol. 127, 949-962 (2001).

33. Moreno, A. A. et al. IRE1/bZIP60-mediated unfolded protein response plays distinct roles in plant immunity and abiotic stress responses. PLoS ONE 7, e31944. https://doi.org/10.1371/journal.pone.0031944 (2012).

34. Martinez, I. M. \& Chrispeels, M. J. Genomic analysis of the unfolded protein response in Arabidopsis shows its connection to important cellular processes. Plant Cell 15, 561-576. https://doi.org/10.1105/tpc.007609 (2003).

35. Lu, S. J. et al. Conservation of IRE1-regulated bZIP74 mRNA unconventional splicing in rice (Oryza sativa L.) involved in ER stress responses. Mol. Plant 5, 504-514. https://doi.org/10.1093/mp/ssr115 (2012).

36. Irsigler, A. S. et al. Expression profiling on soybean leaves reveals integration of ER- and osmotic-stress pathways. BMC Genomics 8, 431. https://doi.org/10.1186/1471-2164-8-431 (2007).

37. Vitale, A. \& Ceriotti, A. Protein quality control mechanisms and protein storage in the endoplasmic reticulum. A conflict of interests?. Plant Physiol. 136, 3420-3426. https://doi.org/10.1104/pp.104.050351 (2004).

38. Ye, C., Dickman, M. B., Whitham, S. A., Payton, M. \& Verchot, J. The unfolded protein response is triggered by a plant viral movement protein. Plant Physiol. 156, 741-755. https://doi.org/10.1104/pp.111.174110 (2011).

39. Deng, Y. et al. Heat induces the splicing by IRE1 of a mRNA encoding a transcription factor involved in the unfolded protein response in Arabidopsis. Proc. Natl. Acad. Sci. USA 108, 7247-7252. https://doi.org/10.1073/pnas.1102117108 (2011).

40. Zhang, S. S. et al. Tissue-specific transcriptomics reveals an important role of the unfolded protein response in maintaining fertility upon heat stress in Arabidopsis. Plant Cell 29, 1007-1023. https://doi.org/10.1105/tpc.16.00916 (2017).

41. Lee, S. C., Choi, H. W., Hwang, I. S., Choi, D. S. \& Hwang, B. K. Functional roles of the pepper pathogen-induced bZIP transcription factor, CAbZIP1, in enhanced resistance to pathogen infection and environmental stresses. Planta 224, 1209-1225. https:// doi.org/10.1007/s00425-006-0302-4 (2006).

42. Deng, Y., Srivastava, R. \& Howell, S. H. Protein kinase and ribonuclease domains of IRE1 confer stress tolerance, vegetative growth, and reproductive development in Arabidopsis. Proc. Natl. Acad. Sci. USA 110, 19633-19638. https://doi.org/10.1073/pnas.13147 49110 (2013).

43. Back, S. H., Schroder, M., Lee, K., Zhang, K. \& Kaufman, R. J. ER stress signaling by regulated splicing: IRE1/HAC1/XBP1. Methods 35, 395-416. https://doi.org/10.1016/j.ymeth.2005.03.001 (2005).

44. Noh, S. J., Kwon, C. S. \& Chung, W. I. Characterization of two homologs of Irelp, a kinase/endoribonuclease in yeast, in Arabidopsis thaliana. Biochim. Biophys. Acta 1575, 130-134. https://doi.org/10.1016/s0167-4781(02)00237-3 (2002).

45. Chen, Y. \& Brandizzi, F. AtIRE1A/AtIRE1B and AGB1 independently control two essential unfolded protein response pathways in Arabidopsis. Plant J. 69, 266-277. https://doi.org/10.1111/j.1365-313X.2011.04788.x (2012).

46. Deng, Y. et al. IRE1, a component of the unfolded protein response signaling pathway, protects pollen development in Arabidopsis from heat stress. Plant J. 88, 193-204. https://doi.org/10.1111/tpj.13239 (2016).

47. Sidrauski, C. \& Walter, P. The transmembrane kinase Irelp is a site-specific endonuclease that initiates mRNA splicing in the unfolded protein response. Cell 90, 1031-1039. https://doi.org/10.1016/s0092-8674(00)80369-4 (1997).

48. Iwata, Y. \& Koizumi, N. An Arabidopsis transcription factor, AtbZIP60, regulates the endoplasmic reticulum stress response in a manner unique to plants. Proc. Natl. Acad. Sci. USA 102, 5280-5285. https://doi.org/10.1073/pnas.0408941102 (2005).

49. Nagashima, Y. et al. Arabidopsis IRE1 catalyses unconventional splicing of bZIP60 mRNA to produce the active transcription factor. Sci. Rep. 1, 29. https://doi.org/10.1038/srep00029 (2011).

50. Hollien, J. et al. Regulated Ire1-dependent decay of messenger RNAs in mammalian cells. J. Cell Biol. 186, 323-331. https://doi. org/10.1083/jcb.200903014 (2009).

51. Maurel, M., Chevet, E., Tavernier, J. \& Gerlo, S. Getting RIDD of RNA: IRE1 in cell fate regulation. Trends Biochem. Sci. 39, 245-254. https://doi.org/10.1016/j.tibs.2014.02.008 (2014).

52. Mishiba, K. et al. Defects in IRE1 enhance cell death and fail to degrade mRNAs encoding secretory pathway proteins in the Arabidopsis unfolded protein response. Proc. Natl. Acad. Sci. USA 110, 5713-5718. https://doi.org/10.1073/pnas.1219047110 (2013).

53. Barnabas, B., Jager, K. \& Feher, A. The effect of drought and heat stress on reproductive processes in cereals. Plant Cell Environ. 31, 11-38. https://doi.org/10.1111/j.1365-3040.2007.01727.x (2008).

54. Zinn, K. E., Tunc-Ozdemir, M. \& Harper, J. F. Temperature stress and plant sexual reproduction: uncovering the weakest links. J. Exp. Bot. 61, 1959-1968. https://doi.org/10.1093/jxb/erq053 (2010).

55. Liu, Y. \& Chang, A. Heat shock response relieves ER stress. EMBO J. 27, 1049-1059. https://doi.org/10.1038/emboj.2008.42 (2008).

56. Xu, X., Gupta, S., Hu, W., McGrath, B. C. \& Cavener, D. R. Hyperthermia induces the ER stress pathway. PLoS ONE 6, e23740. https://doi.org/10.1371/journal.pone.0023740 (2011).

57. Gao, H., Brandizzi, F., Benning, C. \& Larkin, R. M. A membrane-tethered transcription factor defines a branch of the heat stress response in Arabidopsis thaliana. Proc. Natl. Acad. Sci. USA 105, 16398-16403. https://doi.org/10.1073/pnas.0808463105 (2008).

58. Parra-Rojas, J., Moreno, A. A., Mitina, I. \& Orellana, A. The dynamic of the splicing of bZIP60 and the proteins encoded by the spliced and unspliced mRNAs reveals some unique features during the activation of UPR in Arabidopsis thaliana. PLoS ONE 10, e0122936. https://doi.org/10.1371/journal.pone.0122936 (2015). 
59. Liu, X., Rockett, K. S., Kørner, C. J. \& Pajerowska-Mukhtar, K. M. Salicylic acid signalling: new insights and prospects at a quartercentury milestone. Essays Biochem. 58, 101-113 (2015).

60. Boatwright, J. L. \& Pajerowska-Mukhtar, K. Salicylic acid: an old hormone up to new tricks. Mol. Plant Pathol. 14, 623-634 (2013).

61. Vlot, A. C., Dempsey, D. A. \& Klessig, D. F. Salicylic acid, a multifaceted hormone to combat disease. Annu. Rev. Phytopathol. 47, 177-206. https://doi.org/10.1146/annurev.phyto.050908.135202 (2009).

62. Delaney, T. P., Friedrich, L. \& Ryals, J. A. Arabidopsis signal transduction mutant defective in chemically and biologically induced disease resistance. Proc. Natl. Acad. Sci. USA 92, 6602-6606. https://doi.org/10.1073/pnas.92.14.6602 (1995).

63. Alhoraibi, H., Bigeard, J., Rayapuram, N., Colcombet, J. \& Hirt, H. Plant Immunity: the MTI-ETI Model and Beyond. Curr. Issues Mol. Biol. 30, 39-58. https://doi.org/10.21775/cimb.030.039 (2019).

64. Mukhtar, M. S., McCormack, M. E., Argueso, C. T. \& Pajerowska-Mukhtar, K. M. Pathogen tactics to manipulate plant cell death. Curr. Biol. 26, R608-R619. https://doi.org/10.1016/j.cub.2016.02.051 (2016).

65. Hayashi, S., Wakasa, Y. \& Takaiwa, F. Functional integration between defence and IRE1-mediated ER stress response in rice. Sci. Rep. 2, 670. https://doi.org/10.1038/srep00670 (2012).

66. Nagashima, Y., Iwata, Y., Ashida, M., Mishiba, K. \& Koizumi, N. Exogenous salicylic acid activates two signaling arms of the unfolded protein response in Arabidopsis. Plant Cell Physiol. 55, 1772-1778. https://doi.org/10.1093/pcp/pcul08 (2014).

67. Kilian, J. et al. The AtGenExpress global stress expression data set: protocols, evaluation and model data analysis of UV-B light, drought and cold stress responses. Plant J. 50, 347-363. https://doi.org/10.1111/j.1365-313X.2007.03052.x (2007).

68. Lempe, J. et al. Diversity of flowering responses in wild Arabidopsis thaliana strains. PLoS Genet 1, 109-118. https://doi.org/10.1371/ journal.pgen.0010006 (2005).

69. Waese, J. et al. ePlant: visualizing and exploring multiple levels of data for hypothesis generation in plant biology. Plant Cell 29, 1806-1821. https://doi.org/10.1105/tpc.17.00073 (2017).

70. Winter, D. et al. An "Electronic Fluorescent Pictograph" browser for exploring and analyzing large-scale biological data sets. PLoS ONE 2, e718. https://doi.org/10.1371/journal.pone.0000718 (2007).

71. Pajerowska-Mukhtar, K. M. et al. The HSF-like transcription factor TBF1 is a major molecular switch for plant growth-to-defense transition. Curr. Biol. 22, 103-112. https://doi.org/10.1016/j.cub.2011.12.015 (2012).

72. McCormack, M. E., Liu, X., Jordan, M. R. \& Pajerowska-Mukhtar, K. M. An improved high-throughput screening assay for tunicamycin sensitivity in Arabidopsis seedlings. Front. Plant Sci. 6, 663. https://doi.org/10.3389/fpls.2015.00663 (2015).

73. Ohta, M. et al. Analysis of rice ER-resident J-proteins reveals diversity and functional differentiation of the ER-resident Hsp70 system in plants. J. Exp. Bot. 64, 5429-5441. https://doi.org/10.1093/jxb/ert312 (2013).

74. Hong, Z. H., Qing, T., Schubert, D., Kleinmanns, J. A. \& Liu, J. X. BLISTER-regulated vegetative growth is dependent on the protein kinase domain of ER stress modulator IRE1A in Arabidopsis thaliana. PLoS Genet. 15, e1008563. https://doi.org/10.1371/journ al.pgen.1008563 (2019).

75. Yamamoto, M. et al. ERdj3B-mediated quality control maintains anther development at high temperatures. Plant Physiol. 182, 1979-1990. https://doi.org/10.1104/pp.19.01356 (2020).

76. Schott, A. et al. Arabidopsis stromal-derived Factor2 (SDF2) is a crucial target of the unfolded protein response in the endoplasmic reticulum. J. Biol. Chem. 285, 18113-18121. https://doi.org/10.1074/jbc.M110.117176 (2010).

77. Nekrasov, V. et al. Control of the pattern-recognition receptor EFR by an ER protein complex in plant immunity. EMBO J. 28, 3428-3438. https://doi.org/10.1038/emboj.2009.262 (2009).

78. Wang, D., Amornsiripanitch, N. \& Dong, X. A genomic approach to identify regulatory nodes in the transcriptional network of systemic acquired resistance in plants. PLoS Pathog. 2, e123. https://doi.org/10.1371/journal.ppat.0020123 (2006).

79. Pajerowska-Mukhtar, K. M., Emerine, D. K. \& Mukhtar, M. S. Tell me more: roles of NPRs in plant immunity. Trends Plant Sci. 18, 402-411. https://doi.org/10.1016/j.tplants.2013.04.004 (2013).

80. Sun, Y., Detchemendy, T. W., Pajerowska-Mukhtar, K. M. \& Mukhtar, M. S. NPR1 in JazzSet with pathogen effectors. Trends Plant Sci. 23, 469-472. https://doi.org/10.1016/j.tplants.2018.04.007 (2018).

81. Liu, X. et al. Bacterial leaf infiltration assay for fine characterization of plant defense responses using the Arabidopsis thalianaPseudomonas syringae pathosystem. J Vis Exp https://doi.org/10.3791/53364 (2015).

82. Noh, S. J., Kwon, C. S., Oh, D. H., Moon, J. S. \& Chung, W. I. Expression of an evolutionarily distinct novel BiP gene during the unfolded protein response in Arabidopsis thaliana. Gene 311, 81-91. https://doi.org/10.1016/s0378-1119(03)00559-6 (2003).

83. Howell, S. H. When is the unfolded protein response not the unfolded protein response?. Plant Sci. 260, 139-143. https://doi. org/10.1016/j.plantsci.2017.03.014 (2017).

84. Liu, X., Afrin, T. \& Pajerowska-Mukhtar, K. M. Arabidopsis GCN2 kinase contributes to ABA homeostasis and stomatal immunity. Commun. Biol. 2, 302. https://doi.org/10.1038/s42003-019-0544-x (2019).

85. 86Mangano, S., Gonzalez, C. D. \& Petruccelli, S. in Arabidopsis Protocols (eds Jose J. Sanchez-Serrano \& Julio Salinas) 165-173 (Humana Press, 2014).

86. Jefferson, R. A., Kavanagh, T. A. \& Bevan, M. W. GUS fusions: beta-glucuronidase as a sensitive and versatile gene fusion marker in higher plants. EMBO J. 6, 3901-3907 (1987).

\section{Acknowledgements}

We thank Dr. Shahid Mukhtar for editing and critically reading the manuscript, and Mr. Bharat Mishra for assistance with bioinformatics-aided promoter analyses.

\section{Author contributions}

T.A. performed all the experiments described in this study, analysed the data, prepared all figures, and helped with manuscript preparation. M.S. assisted with sample preparation and processing. B.C.T. performed preliminary experiments to confirm the genetic variation of IRE1a in different accessions. K.P.M conceived the project, coordinated the research program, and wrote the manuscript.

\section{Funding}

This work was supported by the National Science Foundation CAREER award (IOS-1350244) to K.P.M., and UAB Blazer and Alabama NSF-EPSCoR GRSP Doctoral Fellowships to T.A.

\section{Competing interests}

The authors declare no competing interests.

Additional information

Supplementary information is available for this paper at https://doi.org/10.1038/s41598-020-76114-1. 
Correspondence and requests for materials should be addressed to K.M.P.-M.

Reprints and permissions information is available at www.nature.com/reprints.

Publisher's note Springer Nature remains neutral with regard to jurisdictional claims in published maps and institutional affiliations.

(c) (i) Open Access This article is licensed under a Creative Commons Attribution 4.0 International cc) License, which permits use, sharing, adaptation, distribution and reproduction in any medium or format, as long as you give appropriate credit to the original author(s) and the source, provide a link to the Creative Commons licence, and indicate if changes were made. The images or other third party material in this article are included in the article's Creative Commons licence, unless indicated otherwise in a credit line to the material. If material is not included in the article's Creative Commons licence and your intended use is not permitted by statutory regulation or exceeds the permitted use, you will need to obtain permission directly from the copyright holder. To view a copy of this licence, visit http://creativecommons.org/licenses/by/4.0/.

(C) The Author(s) 2020 